\title{
Accurate Strain Field Measurement During Strip Rolling by Exploiting Recurring Material Motion with Time-Integrated Digital Image Correlation
}

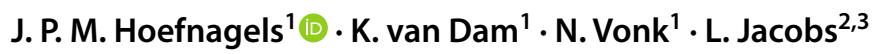

Received: 17 May 2021 / Accepted: 13 September 2021 / Published online: 29 December 2021

(c) The Author(s) 2021

\begin{abstract}
Background 95\% Of all metals and alloys are processed using strip rolling, explaining the great number of existing strip rolling optimization models. Yet, an accurate in-situ full-field experimental measurement method of the deformation, velocity and strain fields of the strip in the deformation zone is lacking.

Objective Here, a novel time-Integrated Digital Image Correlation (t-IDIC) framework is proposed and validated that fully exploits the notion of continuous, recurring material motion during strip rolling.

Methods High strain accuracy and robustness against unavoidable light reflections and missing speckles is achieved by simultaneously correlating many (e.g. 200) image pairs in a single optimization step, i.e. each image pair is correlated with the same average global displacement field but is multiplied by a unique velocity corrector to account for differences in material velocity between image pairs.

Results Demonstration on two different strip rolling experiments revealed previously inaccessible subtle changes in the deformation and strain fields due to minor variations in pre-deformation, elastic recovery, and geometrical irregularities. The influence of the work roll force and entry/exit strip tension has been investigated for strip rolling with an industrial pilot mill, which revealed unexpected non-horizontal material feed. This asymmetry was reduced by increasing the entry strip tension and rolling force, resulting in a more symmetric strain distribution, while increased distance between the neutral and entry point was found for a larger rolling force.

Conclusions The proposed t-IDIC method allows for robust and accurate characterization of the strip's full-field behavior of the deformation zone during rolling, revealing novel insights in the material behavior.
\end{abstract}

Keywords Digital Image Correlation (DIC) · Integrated Digital Image Correlation (IDIC) · Strip rolling · Strain field · Velocity field

\section{Introduction}

Cold rolling is the most important processes considering metal forming. Over $95 \%$ of non-ferrous/ ferrous metals and alloys are processed by strip rolling into different shapes,

J. P. M. Hoefnagels

j.p.m.hoefnagels@tue.nl

1 Department of Mechanical Engineering, Eindhoven University of Technology, Eindhoven, The Netherlands

2 Tata Steel, Research \& Development, IJmuiden, The Netherlands

3 Faculty of Engineering Technology, University of Twente, Enschede, The Netherlands i.e. plates, strips, sheets, bars, rods, etc. During the rolling process, the material is heavily compressed by two rotating cylindrical rolls resulting in permanent deformation of the strip. The different-shaped end product can be used for, e.g. automotive parts, packaging, construction and yellow (construction)/ white (household-products) goods [1-3]. Furthermore, cold rolling allows to increase the strength of the metal up to $400 \%$ due to strain hardening, while it improves the surface finish and provides stricter dimensional tolerances $[2,4,5]$.

Generally, a tandem mill consisting of several rolling stands is used in strip rolling to achieve large thickness reductions. In each stand, the thickness of the strip is reduced by a certain amount, depending on the processing conditions, i.e. the work roll force, the entry and exit strip 
tension, the entrance velocity, etc. High industrial interest has led researchers to develop models that are used to better predict, analyze and understand the rolling process $[3$, 4, 6-11]. However, most of these models don't take into account some key phenomena that are known to occur in the deformation zone: (i) pre-deformation in the material before it first touches the work roll, (ii) elastic recovery (spring-back) after the strip is processed, (iii) the gradient in strip velocity over the strip thickness. As a result these phenomena are neglected in the prediction of some key output parameters of the models, i.e. the change in contact length, neutral point (i.e. the position at the edge where the strip velocity equals the work roll velocity) and entry and exit point (i.e. the point where the strip, respectively, first and last touches the work roll), each depicted, with some other significant rolling parameters, in Fig. 1. Without taking into account these phenomena, the strip rolling process in the deformation zone can never be fully understood, predicted and optimized. Therefore, there is a continuous demand to further improve existing models. To achieve this, it would be of great benefit if, by means of experiments, the full velocity and strain fields in the deformation zone between the work rolls could be accurately measured, which then could be studied as a function of the various input parameters, e.g., the rolling force and strip tension. Additionally, this data would allow direct identification of key strip rolling characteristics, including the neutral, entry, and exit point, and volume contraction.

Efforts in direct acquisition of full-field data from the strip rolling process is, however, seriously obstructed by multiple factors: (i) the harsh environment in which the steel strip is processed, i.e. oil contamination and machine vibrations, (ii) the obstructed view and poor lightning conditions of the rolling process due to very limited space to place cameras and

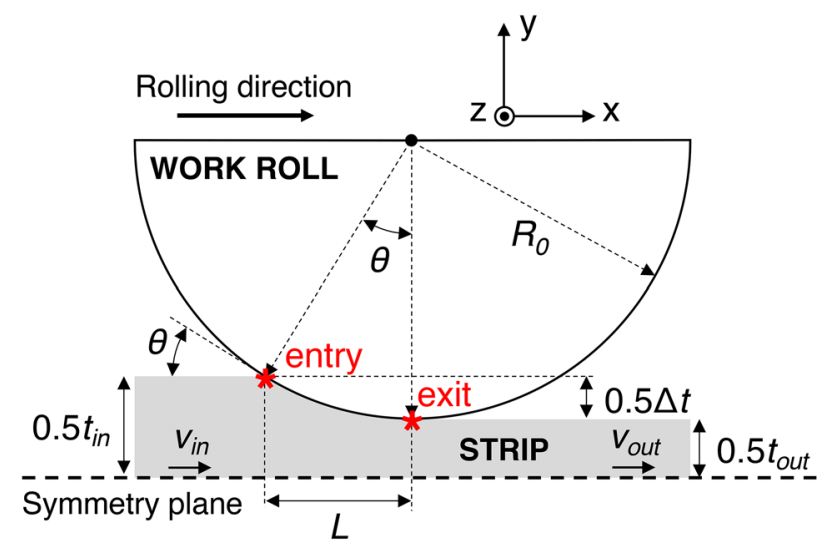

Fig. 1 Schematic of the strip rolling process, with the significant rolling parameters: contact angle $(\theta)$ and length $(L)$, work roll radius $\left(R_{0}\right)$, inlet $\left(t_{\text {in }}\right)$, outlet $\left(t_{\text {out }}\right)$ and reduction thickness $(\Delta t)$, and inlet $\left(v_{\text {in }}\right)$ and outlet strip velocity $\left(v_{\text {out }}\right)$ illumination in between the rolls, (iii) the high material velocity requiring a short shutter time and hence increased acquisition noise, (iv) inevitable reflections of light on the surface of the rolls and the strip, combined with (v) the difficulty to apply even a reasonable quality speckle pattern, in combination with approximately periodic vertical grooves on the sides of the strip originating from the coil slitting process (cutting to specific width), thereby seriously hampering standard particle or pattern tracking methods. Figure 2, shows the (optimized) pattern quality of a steel strip processed in a large-scale pilot mill, where the above-mentioned complications can clearly be seen. As a result the image quality is much lower than typically used in full-field measurements. As these complications seem unavoidable, a highly stable method needs to be developed that is sufficiently robust to accurately correlate poor quality images, as shown in Fig. 2(b), to obtain meaningful full-field data of the deformation zone.

Over the years, various image processing methods have been developed to obtain full-field displacement, velocity and/or strain data. Particle tracking velocimetry (PTV) is a widely used method in fluid mechanics to measure, from consecutive images of a flow, the individual trajectory and velocity of small tracer particles that are assumed to perfectly follow the flow dynamics [12]. However, as the particle density is controlled by the flow and is thus typically (highly) in-homogenous, difficulties arise in converting the particle trajectories in a continuous velocity field. Therefore, nowadays, particle image velocimetry (PIV) is more widely employed for flow visualization (from an image series), with the difference being that the tracer particle density is significantly increased to the point that tracing of individual particles is no longer possible. Instead, regions (interrogation windows) containing many particles are being traced to obtain a full vector field [13]. In the field of hydrodynamics, where displacements are large and the interest lies in the $2 \mathrm{D}$ or $3 \mathrm{D}$ velocity field, it is sufficiently accurate to

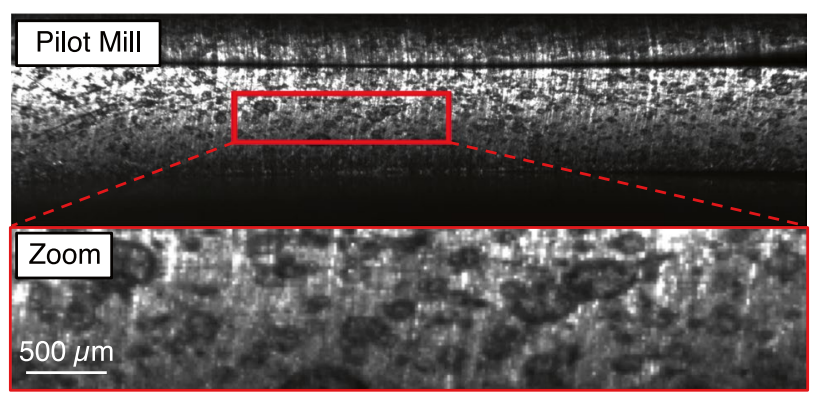

Fig. 2 Image quality of a $2.4 \mathrm{~mm}$ thick steel strip processed through a pilot mill at a rolling velocity of $10 \mathrm{~mm} / \mathrm{s}$ with zoomed view of the non-ideal pattern. Note that significant effort has been put in to optimizing the pattern quality, camera settings and illumination conditions. The periodic vertical grooves originate from the coil slitting process during which the strip is cut to a specific width. 
trace the interrogation windows by optimizing only the rigid body displacement, while neglecting deformations inside the windows. In contrast, within the field of solid mechanics, the interest lies in determination of the full strain fields, with the strain magnitude often only a few percent, corresponding to sub-pixel displacements in the consecutive images. In that case, it was found that not only the position but also the deformation of the interrogation windows should be optimized, which is done in what has become known as local Digital Image Correlation (local DIC), for which many commercial software packages exist. This difference is, e.g., nicely illustrated in [14], where PIV was used to measure the hydrodynamic load on a flexible plate from the velocity field of the surrounding flow, while local DIC was used to measure the deformation of the flexible plate. In a way, the material kinetics in strip rolling can be regarded as in between a stiff island and its surrounding fluid flow, because the solid strip heavily deforms between the rolls exhibiting large plastic flow, but nevertheless highly accurate strain measurement is required to confront existing strip rolling models to (small) errors in, e.g., the volumetric change, neutral point and entry and exit points. As the accuracy of PTV and PIV is clearly insufficient for this purpose, Li et al. [15] used DIC to try to observe the complete rolling process and managed to obtain several rolling characteristics, particularly the forward and backward slip, neutral plane and neutral angle with reasonable accuracy. However, the regular local DIC algorithm that was used must have been seriously challenged by the abovementioned five complication factors for in-situ full-field measurements of strip rolling, which may explain why only the displacement fields but no strain fields were reported. To the best of the authors' knowledge no further study of DIC applied on a strip rolling process has been reported.

The first step to make the DIC algorithm more robust is by utilizing knowledge on the mechanics of the problem. Instead of separate correlation of many small interrogation windows, as done in local DIC, so-called "global DIC" (GDIC) [16] uses the fact that the material remains continuous (e.g. during strip rolling) to correlate the complete region of interest (ROI). Continuity is imposed by describing the displacement field by means of a kinematic regularization (e.g. a polynomial basis) with a reduced amount of degrees of freedom (DOFs). The increased ratio of the number of measurements (i.e. the light intensity value at each pixel) to the number of variables (DOFs) makes a GDIC algorithm more robust against pattern degradation (e.g. due to surface reflections) and high acquisition noise [17-20], which are unavoidable in rolling processes as shown in Fig. 2. GDIC has been successfully applied to various micro-mechanical problems, each with their own challenges, by fine-tuning the choice of kinematic regularization to achieve optimal robustness and accuracy in the displacement and strain field [21-25]. Specifically note-worthy is the fine tuning done in [26-28], where time-Integrated Digital Image
Correlation algorithms are proposed exploiting the periodicity of the deformation to simultaneously correlate all images at once to stabilize the correlation and identify material parameters with high sensitivity, by further increasing the ratio of measurement points to DOFs. Where in [27] this algorithm was only validated on synthetically generated images, the strength of this method was later demonstrated on experimental images, for instance for the challenging test case of identifying insensitive mixed-mode interface adhesion properties [29, 30]. In [28], this concept was again proven to be valuable for vibrational analysis, while in an earlier more specific paper a time-integrated algorithm tuned to periodic motion has been validated for fatigue testing [26]. These works show that by significantly increasing amount of measured values for the same low amount of DOFs, the accuracy and stability is improved. Therefore, designing a time-integrated (global) DIC algorithm that is dedicated and fine-tuned to the strip rolling process seems to be the best candidate to achieve optimal accuracy in the velocity and strain fields in the deformation zone. In turn, this may enable successful identification of all key rolling parameters, i.e. the entry, exit, and neutral point, volumetric contraction/expansion, velocity gradients, etc. which would enable accurate validation of the analytical rolling models.

The goal of this work is to develop a complete methodology to measure velocity and strain fields during strip rolling by designing a time-integrated DIC framework that fully exploits the knowledge of recurring material motion (and material continuity) in a simultaneous correlation of many image pairs (Section 2). With this new t-DIC framework in place, a dedicated optical camera with lighting setup is developed to record images that are the least hampered by the obstructed view and the harsh environment (Section 3). With this setup, images have been recorded of two very different types of strip rolling processes: (i) an easy-accessible smallscale hand mill in which a rubber strip is processed used for algorithm validation purposes and (ii) a large-scale 'pilot mill' in which a steel strip is processed, for which the view is highly obstructed and the harsh environment applies. The results of a range of validation measurements are discussed in detail in Section 4. Two proof-of-principle test cases, i.e. (i) varying the work roll forces and (ii) varying entry and exit strip tensions are presented and discussed in Section 5, after which the general conclusions are given (Section 6).

\section{Derivation of a Time-Integrated Digital Image Correlation Framework for Recurring Material Motion}

The novel t-IDIC framework that is fine-tuned for recurring material motion during strip rolling and based on multiple image pairs is presented here. This method is based on global DIC (GDIC), therefore, first the general GDIC approach is 
shortly explained. Subsequently, the adaptations are elaborated that enable robust and accurate correlation of recurring material motion, which is done by extending the GDIC framework to make it time-integrated (t-IDIC). Finally, the associated post-processing steps are discussed. The complete rigorous derivation of the t-IDIC framework and its system of equations, following the one-step linearization of the brightness conservation equation as proposed by Neggers et al. [16], is presented in 7.

\section{Global Digital Image Correlation}

Contrary to local DIC in which multiple local ROIs (also named subsets or windows) are correlated independently, in GDIC a global ROI that is often (nearly) as large as the complete field of view (FOV) is correlated in a single optimization step. Moreover, in most experiments the material behaves as a continuum during deformation. Therefore, the condition of material continuity is intrinsically imposed in GDIC (over the global ROI), which makes the correlation problem more constrained and consequently more robust against acquisition noise compared to local DIC. As a result, GDIC has the potential to be more accurate, however, only under the condition that a kinematic basis is chosen that is sufficiently rich to be able to describe the true deformation field that occurs in the experiment. Note that even for a rich kinematic basis the number of DOFs is much less than that of local DIC where many subsets are needed to cover the complete FOV $[17,18]$. As a consequence, global correlation is generally favorable when the material is known to behave as a continuum, especially when large localized gradients in the deformation are absent [31], as is precisely the case for strip rolling processes. Consequently, a global approach is chosen for the here-presented method.

As for all DIC approaches, GDIC is based on brightness conservation, i.e. the brightness of each material point should stay constant throughout the deformation process, leading to:

$$
\begin{array}{r}
f(\underline{x}) \approx g \circ \underline{\Phi}(\underline{x}), \\
\underline{\Phi}(\underline{x})=\underline{x}+\underline{U}(\underline{x}),
\end{array}
$$

in which $f$ and $g$ are the image of, respectively, the reference and deformed state, which are gray-scale intensity fields, $\underline{\Phi}(\underline{x})$ describes the mapping function for the transformation of the reference coordinates $\underline{x}$ to the deformed coordinates based on the true displacement field $\underline{U}(\underline{x})$, and the symbol o denotes the composition of two functions, i.e. $(g \circ \underline{\Phi})(\underline{x})=g(\underline{\Phi}(\underline{x}))$. It should be noted that the pixel positions $\underline{x}$ are all fixed and refer back to the first reference image, as is the case for the displacement field, i.e. all fields are described in a Lagrangian frame of reference.

The problem of finding the true displacement field that satisfies equation (1) is impossible to solve, because this system is ill-posed with twice as much unknowns (DOFs) than knowns, i.e. two unknown displacement vector components per pixel versus one known measured light intensity value per pixel. To circumvent this issue, all DIC methods use some sort of approximation of the true displacement field (in a process called 'regularization') by describing the displacement field with a reduced number of DOFs,

$$
\begin{aligned}
& \underline{U}(\underline{x}) \approx \underline{u}(\underline{x}, \underset{\sim}{\lambda}, \\
& \underline{\Phi}(\underline{x}) \approx \underline{\phi}(\underline{x}, \underset{\sim}{\lambda},
\end{aligned}
$$

in which $\underline{u}(\underline{x}, \lambda)$ is the approximated, parameterized displacement field, $\lambda$ is an array containing the DOFs, $\underset{\sim}{\lambda}=\left[\lambda_{1}, \lambda_{2}, \ldots ., \tilde{\lambda}_{n}\right]^{T}$, and $\left.\underline{\phi} \underset{\sim}{x} \underset{\sim}{\lambda}\right)$ is the approximated mapping function. Then the brightness conservation equation (1) can be approximated by,

$$
\begin{array}{r}
f(\underline{x}) \approx g \circ \underline{\phi} \underline{(x,} \underset{\sim}{\lambda}, \\
\underline{\phi}(\underline{x}, \underset{\sim}{\lambda})=\underline{x}+\underline{u} \underline{x} \underset{\sim}{\lambda} .
\end{array}
$$

Often the choice of regularization simply consists of parameterizating the displacement field with a linearly independent kinematic basis that is multiplied by the DOFs, $\lambda$. For instance, a basis of polynomial shape functions could be used:

$$
\begin{aligned}
\underline{u}(\underline{x}, \underset{\sim}{\lambda}) & =u_{x}(\underset{\sim}{x} \underset{\sim}{\lambda}) \cdot \underline{e}_{x}+u_{y}(\underset{x}{\underline{x}} \underset{\sim}{\lambda}) \cdot \underline{e}_{y} \\
& =\sum_{i=0}^{n} \sum_{j=0}^{m} \lambda_{x i j} x^{i} y^{j} \underline{e}_{x}+\sum_{i=0}^{n} \sum_{j=0}^{m} \lambda_{y i j} x^{i} y^{j} \underline{e}_{y} .
\end{aligned}
$$

The larger the ratio of pixels per DOF, the more the detrimental effect of image noise is suppressed, therefore, the kinematic basis should be chosen sufficiently 'rich' but not more than that.

The search for the displacement field then consists in finding the optimal DOFs, $\lambda^{\text {opt }}$, for which the approximated displacement field, $\underline{u}\left(\underline{x}, \lambda^{o \tilde{p t} t}\right)$, best satisfies the brightness conservation over the global ROI, here denoted with $\Omega$. In practice, this problem is cast in terms of the minimization of a cost function, $\Psi(\underset{\sim}{\lambda})$, that describes the (spatially averaged) brightness conservation violation:

$$
\begin{aligned}
\Psi(\underset{\sim}{\lambda}) & =\int_{\Omega} \frac{1}{2}\left[r(\underline{x}, \underset{\sim}{\lambda}]^{2} d \underline{x}\right. \\
& \approx \sum_{\Omega_{s}} \frac{1}{2}[f(\underline{x})-g \circ \underline{\phi} \underline{x} \underset{\sim}{x} \underset{\sim}{\lambda}]^{2},
\end{aligned}
$$

where $r(\underline{x}, \lambda)=f(\underline{x})-g \circ \underline{\phi}(\underline{x}, \underline{\lambda})$ is the image residual, which should ideally be zero for $\bar{\lambda}=\tilde{\sim} \lambda^{\text {opt }}$ in the absence of image acquisition noise and numerical errors, such as interpolation errors [32]. Therefore, $\underset{\sim}{\lambda^{o p t}}$ is found by minimization of $\Psi(\underset{\sim}{\lambda})$ : 


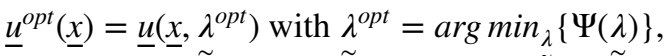

which completes the system of equations.

Finally, the solution to this non-linear minimization problem may be found by means of a (modified) Gauss-Newton iterative optimization scheme that can be easily solved numerically. A rigorous derivation of this (modified) GaussNewton scheme, which follows from a one-step linearization of the non-linear brightness conservation equation, is given in the work of Neggers et al. [16]. The GDIC framework is summarized on the left hand side of Table 1, where it is compared to the t-IDIC framework that is discussed next.

\section{Extension to a Novel Time-Integrated Digital Image Correlation Framework}

GDIC is based on correlating each deformed image to a single, undeformed reference image, which means that a separate correlation is performed for each image. However, the case of strip rolling is special as the flow of material is not only continuous but also recurring, therefore, the incremental deformation field between consecutive images should be constant over time. This provides us with the possibility to not correlate each image separately but, instead, correlate many pairs of consecutive images simultaneously, here called 'image pairs', using a single parameterized incremental displacement field, $\delta u_{a v}$. In general, when physical knowledge of the deformation process is utilized in the image correlation operation, the correlation process is called integrated DIC. Here, the knowledge of recurring material motion during strip rolling is utilized in order to simultaneously correlate a set of multiple images, captured over time, therefore, this is best described as a time-integrated DIC (t-IDIC) framework. Important to realize is that the magnitude of the incremental deformation field between each image pair will never be exactly the same because of small variations in (i) the rolling velocity and (ii) the image capture time for a regular high-speed camera (i.e. one that is not triggered externally by means of a precise trigger). To cope with these variations, the averaged incremental deformation field, $\delta u_{a v}$, is multiplied with a scalar 'velocity corrector' $\alpha_{t}$ that is unique for each image pair; this adds one extra DOF per image pair. Please note that the goal here is to find $\delta u_{a v}$, the velocity corrector is only added to improve the correlation's outcome. Consequently, the incremental displacement field for each image pair, $\delta u_{t}$, at time instance $t$ can be written as,

$\underline{\delta u}_{t}\left(\underline{x}_{\sim_{s}}^{\lambda}, \alpha_{t}\right)=\alpha_{t} \underline{\delta u}_{a v}\left(\underset{\sim}{x} \underset{\sim_{s}}{\lambda}\right)$

and Equation (3) is adapted to

$$
\begin{aligned}
& g_{t-1}(\underline{x}) \approx g_{t}(\underline{x}) \circ \underline{\phi}_{t}\left(\underset{x}{x} \underset{\sim}{\lambda}, \alpha_{t}\right), \\
& \underline{\phi}_{t}\left(\underset{x}{x} \underset{\sim s}{\lambda}, \alpha_{t}\right)=\underline{x}+\alpha_{t} \underline{\delta u}_{a v}(\underset{\sim}{x} \underset{\sim}{\lambda}),
\end{aligned}
$$

where the approximated mapping function, $\underline{\phi}_{t}\left(\underline{x}_{\sim_{s}}^{\lambda}, \alpha_{t}\right)$, at each time instance, depends on all the spatial DOFs, $\underset{\sim_{s}}{\lambda}$, and one unique time-related velocity corrector, $\alpha_{t}$. All DOFS are stacked together as $\underset{\sim}{\lambda}=\left[\underset{\sim_{s}}{\lambda} \underset{\sim}{\alpha}\right]^{T}$, in which the relation between the spatial, temporal, and combined DOFs is defined by, respectively, set $S=\left\{n: 1 \leq n \leq s_{D O F}\right\}$, set $T=\left\{n: s_{D O F}+1 \leq n \leq s_{D O F}+t_{D O F}\right\}$, and set $N=\{n$ : $\left.1 \leq n \leq n_{D O F}\right\}=S \cup T$, with $s_{D O F}, t_{D O F}$ and $n_{D O F}$ being, respectively, the number of spatial, temporal, and combined DOFs. Please note that the for the first image pair, $\alpha_{t}$ is set to 1 , therefore, $t_{D O F}$ equals the number of image pairs minus 1. The problem of finding the incremental displacement field, $\underline{\delta u}_{a v}(\underline{x} \underset{\sim s}{\lambda, \alpha}, \underset{\sim}{\alpha})$, is now cast as:

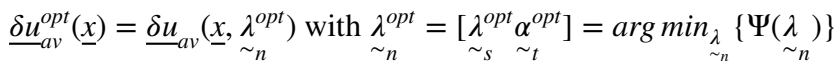

where the cost function, $\Psi$, of the non-linearized brightness conservation is given as,

Table 1 the Global Digital Image Correlation (GDIC) framework compared to the here-proposed time-Integrated Digital Image Correlation (t-IDIC) framework for recurring material motion. For both frameworks, the system of equations is closed with the choice for kinematic regu-

\begin{tabular}{|c|c|}
\hline GDIC & t-IDIC for recurring material motion \\
\hline${\stackrel{\sim}{\lambda^{o p t}}}^{\arg \min } \underset{\sim}{\lambda}\{\Psi(\underset{\sim}{\lambda})\}$ & $\underset{\sim}{\lambda_{n}^{o p t}}=\left[\underset{\sim_{s}}{\lambda_{o}^{o p t}} \underset{\sim}{\alpha_{t}^{o p t}}\right]^{T}=\underset{\sim_{n}}{\arg \min }\{\Psi(\underset{\sim}{\lambda})\}$ \\
\hline$\Psi(\underset{\sim}{\lambda})=\sum_{\Omega_{s}}^{N p x} \frac{1}{2}[f(\underline{x})-g \circ \underline{\phi}(\underline{x}, \underset{\sim}{\lambda})]^{2}$ & $\Psi \underset{\sim}{(\lambda})=\sum_{t=1}^{t_{d o f}} \sum_{\Omega_{s}}^{N p x} \frac{1}{2}\left[g_{t-1}(\underline{x})-g_{t}(\underline{x}) \circ \underline{\phi}_{t}\left(\underset{\sim}{x}, \underset{\sim}{\lambda}, \alpha_{t}\right)\right]^{2}$ \\
\hline$\underline{\phi}(\underline{x}, \underset{\sim}{\lambda})=\underline{x}+\underline{u}(\underline{x}, \underset{\sim}{\lambda})$ & $\underline{\phi}_{t}\left(\underline{x},{\underset{\sim}{s}}_{s}, \alpha_{t}\right)=\underline{x}+\alpha_{t} \underline{\delta u} \underline{u}_{a v}\left(\underline{x},{\underset{\sim}{s}}_{s}\right)$ \\
\hline e.g.: $\underline{u}=\sum_{\chi=1}^{2} \sum_{i=0}^{n} \sum_{j=0}^{m} \lambda_{\chi i j} x^{i} y^{j} \underline{e}_{\chi}$ & e.g.: $\underline{\delta u}_{a v}=\sum_{\chi=1}^{2} \sum_{i=0}^{n} \sum_{j=0}^{m} \lambda_{s, x i j} x^{i} y^{j} \underline{e}_{\chi}$ \\
\hline
\end{tabular}
larization (bottom row); as an example, here, a basis of polynomial shape functions is selected 


$$
\begin{aligned}
\Psi(\underset{\sim n}{\lambda}) & =\sum_{t=1}^{t_{\text {dof }}} \int_{\Omega} \frac{1}{2}\left[r_{t}(\underline{x}, \underset{\sim}{\lambda})\right]^{2} d \underline{x} \\
& \approx \sum_{t=1}^{t_{\text {dof }}} \sum_{\Omega_{s}}^{N p x} \frac{1}{2}\left[g_{t-1}(\underline{x})-g_{t}(\underline{x}) \circ \underline{\phi} \underset{t}{\left.\left(\underset{x_{s}}{x} \underset{\sim_{s}}{\lambda}, \alpha_{t}\right)\right]^{2} .}\right.
\end{aligned}
$$

The complete system of equations of the here-proposed novel t-IDIC framework for recurring material motion is summarized in Table 1.

As for the GDIC framework, the solution to the nonlinear minimization problem of this t-IDIC framework is found by means of a (modified) Gauss-Newton iterative optimization scheme that can be easily solved numerically. A rigorous derivation of this (modified) Gauss-Newton scheme, following a one-step linearization of the non-linear brightness conservation equation, is given in Appendix A. It is important to realize from equation (7) that, even when a linearly-independent kinematic basis is selected for $\delta u_{a v}$, due to the addition of the velocity correctors, the kinematic basis of $\delta u_{t}$ will never be linearly independent, which results in an additional term, $M^{c}$, in the tangent of the system of equations, as is shown in Appendix A. Note that for all of the following correlations, convergence is found when the change in the mean value of $\delta u_{a v}$ is below $1 \cdot 10^{-5}$ pixel.

Notice that the cost function, $\Psi$, includes the summation over all $t_{D O F}$ experimental images (which could be hundreds of images), which are all simultaneously correlated with a reduced amount of DOFs, $\lambda_{n}$, potentially providing a significant improvement of the robustness and accuracy due to a heavy suppression of the image noise. For instance, in the pilot mill strip rolling example discussed below, a total of 200 images $\left(g_{t}\right)$ are obtained over time and are subsequently correlated using an $8^{\text {th }}$ and $3^{\text {rd }}$ order polynomial basis in, respectively, the length and thickness of the processed strip (this choice is motivated in Section 4). This results in $\underset{\sim n}{\lambda}$ containing, respectively, 198 temporal and 60 spatial degrees of freedom. As will be shown below, for every image, the processed strip is captured in a rectangle of $\sim 1200 \times 110$ pixels, resulting in a total of $1.32 \cdot 10^{5}$ pixels (measurement values) per image. Hence, for this specific example, a total of 2.64 . $10^{7}$ known values are used to determine the 258 unknowns, resulting in a ratio of $1: 102326$. Compare this to a typical ratio of 1 : 52 for local DIC (for a standard $25 \times 25$ subset size, correlated with recommended quadratic deformation of the subset based on 12 DOFs) and $1: 2200$ for regular GDIC (with the same polynomial basis as used above).

\section{Post-Processing and Interpretation}

The t-IDIC framework produces the average incremental displacement field, $\underline{\delta u}_{a v}(\underline{x})$, as output. By dividing this vector field with the time increment between two images (camera's shutter time), the velocity vector field, $\underline{v}(\underline{x})=v_{x}(\underline{x}) \cdot \underline{e}_{x}+v_{y}(\underline{x})$ $\cdot \underline{e}_{y}$, is obtained, of which the horizontal velocity field, $v_{x}(\underline{x})$, allows for identification of the neutral point i.e. the position where the strip velocity equals the work roll velocity.

Alternatively, from the average incremental displacement field, the (average) incremental deformation gradient tensor field between image pairs, $\underline{\underline{\delta F^{2}}}\left(\underline{x}, \underset{\sim_{s}}{\lambda}\right)$, can easily be computed using

$\underline{\underline{\delta F}}^{2 D}=\underline{\underline{I}}+\underline{\underline{\operatorname{grad}}}\left(\underline{\underline{\delta u_{a v}}}\right)^{T}=\underline{\underline{\operatorname{grad}}}(\underline{\underline{\phi}} \underline{\underline{ }})^{T}$,

where $\underline{\phi}_{a v}\left(\underset{\sim}{x}, \lambda_{s}\right)$ is the deformation mapping function corresponding to $\underline{\delta u}_{a v}\left(\underline{x}, \underset{\sim_{s}}{\lambda}\right)$, and where the superscript "2D" has been added to emphasize that it reflects only the in-plane deformation, yielding a two-dimensional (incremental) deformation gradient tensor. In order to track the deformation of a material point as it passes through the rolls, the accumulated, total deformation gradient tensor should be computed. In general, the total deformation gradient tensor can be found as the product of the deformation gradient tensor of individual deformation steps (e.g. $\underline{F}_{t o t}=\underline{F} \cdot \underline{F}_{1}$, where a first deformation step, $d \underline{x}_{1}=\underline{F}_{1} \cdot d \underline{x}_{0}$, is followed by a second deformation step, $d \underline{x}_{2}=\underline{F}_{2} \cdot d \underline{x}_{1}$, yielding $\left.d \underline{x}_{2}=\underline{F}_{\text {tot }} \cdot d \underline{x}_{0}\right)$. In our case, for each deformation step the same incremental deformation gradient tensor should be used, i.e $\underline{F}_{1}^{2 D}(\underline{x})=\underline{F}_{2}^{2 D}(\underline{x})=\ldots=\underline{\underline{\delta F^{2 D}}}(\underline{x})$, which is inhomogeneous, therefore, care should be taken to probe it at the correct, updated position after each deformation step. This results in the following nested multiplication:

$\underline{F}_{t o t}^{2 D} \circ \underline{\phi}_{a v}^{\circ q_{t o t}}(\underline{x})=\prod_{q=1}^{q_{t o t}}\left(\underline{\delta F}^{2 D} \circ \underline{\phi}_{a v}^{\circ q}(\underline{x})\right)$,

where the incremental deformation gradient tensor field is iteratively probed each time at the new updated position (until the material point has passed outside the FOV), and the updated position $\underline{\phi}_{a v}^{\circ q}(\underline{x})=\underline{\phi}_{a v}^{q} \circ \underline{\phi}_{a v}^{q-1} \circ \ldots \circ \underline{\phi}_{a v}^{2} \circ \underline{\phi}_{a v}^{1}(\underline{x})$ is found by $q$ times iteratively probing the same mapping function $\phi_{a v}(\underline{x})$. The left hand side shows that the equation yields $\underline{F}_{t o t}^{2 D}$ at deformed configuration $\underline{\phi}_{a v}^{\circ q_{t o t}}(\underline{x})$. A continuous field of the total deformation gradient tensor over the complete FOV is obtained by tracking a sufficient number of initial material points ('seed points') such that their updated positions, $\underline{\phi}_{a v}^{\circ q_{t o t}}(\underline{x})$, cover each pixel position of the strip between the rolls, after which a regular grid of the total deformation gradient tensor at the pixel positions is obtained by (linear) interpolation. 
Subsequently, the strain tensor field is computed using the Green-Lagrange strain definition, i.e. $\underline{\epsilon}^{t o t}(\underline{x})=\frac{1}{2}\left(\underline{F}_{t o t}^{2 D^{T}}(\underline{x}) \cdot \underline{F}_{t o t}^{2 D}\right.$ $(\underline{x})-\underline{I})$, as this properly accounts for large strains and rotations. The vertical strain field, $\epsilon_{y y}^{t o t}(\underline{x})=\underline{e}_{y} \cdot \underline{\epsilon}^{t o t}(\underline{x}) \cdot \underline{e}_{y}$ shows the magnitude of compression of the processed strip between the rolls, while the horizontal strain field, $\epsilon_{x x}^{t o t}(\underline{x})=\underline{e}_{x} \cdot \underline{\epsilon}^{t o t}$ $(\underline{x}) \cdot \underline{e}_{x}$, denotes the elongation along the strip direction, and the shear strain field, $\epsilon_{x y}^{t o t}(\underline{x})=\underline{e}_{x} \cdot \underline{\epsilon}^{t o t}(\underline{x}) \cdot \underline{e}_{y}$, provides quantitative information on the top/down (a)symmetry of the rolling process. The above-given equations for the GreenLagrange strain can also be used to determine the incremental strain fields when $\underline{\underline{\epsilon}}^{i n c}(\underline{x})=\frac{1}{2}\left(\underline{\underline{\delta F^{2 D}}}{ }^{T}(\underline{x}) \cdot \underline{\underline{\delta F}}{ }^{2 D}(\underline{x})-\underline{I}\right)$ is used,

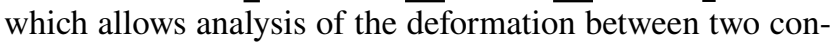
secutive images.

FInally, (the field of) the determinant of the deformation gradient tensor, $\operatorname{det}\left(\underline{F}_{\text {tot }}^{2 D}(\underline{x})\right)$, expresses the surface area ratio given as $A / A_{0}$ that occurs during the rolling process. As plastic deformation is ideally a volume-invariant deformation, $\operatorname{det}\left(\underline{F}_{\text {tot }}(\underline{x})\right)=V / V_{0}$ should only show elastic deformation and thus should ideally be exactly equal to 1 after elastic unloading beyond the work roll's exit, which can thus be used to validate the accuracy of the strain field measurement. Note, however, that $A / A_{0}$ only reflects the change in surface area and not the change in volume as a result for plastically deforming materials $A / A_{0}$, and therefore should be slightly smaller than 1 after elastic unloading because a limited amount of plastic crossexpansion is expected to occur in the direction perpendicular to the FOV, as the compression state between the rolls will never be completely plane strain for the imaged side surface of the strip.

In reflection, all measurands of interest are derived in a large deformation consistent manner, i.e. the velocity vector field results directly from the average incremental displacement field through division by the average time between consecutive images, while the fields of the total strain tensor components and the surface area ratio are derived through the consistent composition of the incremental deformation gradient tensor into the total deformation gradient tensor. Note that the values of the velocity corrector for each image do not affect the average incremental displacement field and are, thus, not used to compute any of these measurands of interest, but they can still be used to monitor the temporal variation in the rolling speed.

\section{Experimental Methodology}

To validate the t-IDIC framework for its capability to produce accurate velocity and strain field of the rolling process, two very different rolling test cases are conducted: (i) a test case of a rubber strip processed with a small-scale hand mill and (ii) a test case of a steel strip processed with a large-scale pilot mill, both shown in Fig. 3. The zoomed views (especially of the pilot mill) clearly show severe image noise, low resolution, and poor contrast, even though the experimental conditions were extensively optimized. Therefore, the method will be first validated on test case (i), where the environment is less harsh, illumination is better, the strip velocity can be changed manually and the ROI of the hand mill has larger height/width ratio which is better suited for DIC. Afterwards the method will be applied to the industrial test case.

\section{Hand Mill}

A rubber strip is processed through a small-scale hand mill as shown in Fig. 3(a), by hand turning a lever connected to the top axle. This mill is only suitable for soft

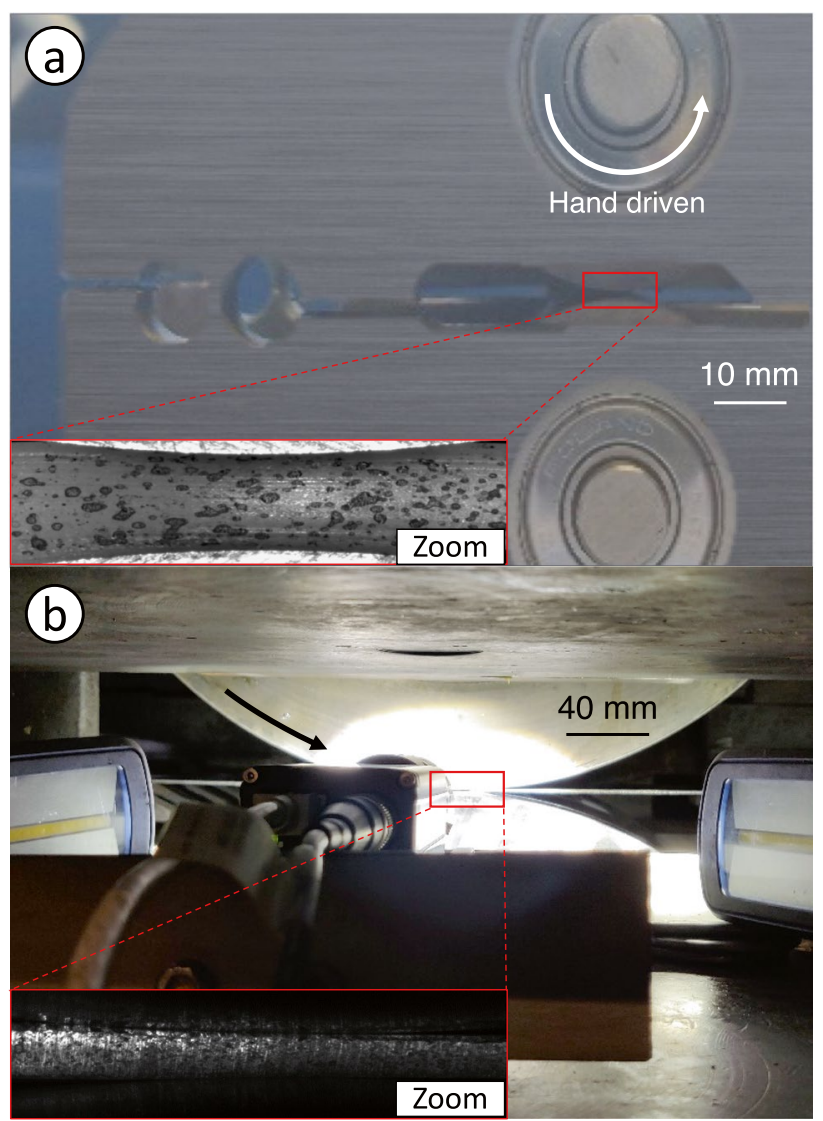

Fig. 3 Experimental setups of the two validation test cases: (a) a lab-scale hand-driven mill with zoom showing the rather convenient optical access to the white rubber strip with speckle pattern and (b) a large-scale pilot mill with a zoomed in view of the processed steel strip with speckle pattern. Whereas the pattern quality, lighting conditions and degree of surface reflection is still quite acceptable for the test case (a), it is clearly problematic for the harsh experimental conditions of test case (b) even though much effort has been invested in optimizing the pattern and image quality (shown in the zoom of (b)) for this test case 
materials, such as ultra-pure, thin metals foils [33] or the $2.5 \mathrm{~mm}$ thick rubber strip deformed in this work, because it is hand driven and the structure is not designed for large forces. Using graphite spray painting, a well-adhered black speckle pattern was applied on the white surface of a rubber strip with initial thickness of $2.5 \mathrm{~mm}$, which was rolled using the hand mill with $40 \mathrm{~mm}$ diameter work rolls, yielding a reduced thickness of $1.87 \mathrm{~mm}$ (note that the rolling force could not be measured in the hand mill). The images were captured using an inexpensive dino-lite-AD7013MTL handheld-digital-microscope fixed and focused on the processed rubber strip. This camera has a 5MP sensor with a working distance of $150 \mathrm{~mm}$, a resolution of $2592 \times 1944$. The frame rate was set to one image per second in according to the hand driving speed to have roughly the same displacement magnitude, in terms of pixels in the image, between two consecutive images as in test case (b). Two light sources are used to illuminate the strip from both sides of the camera, similar to the camera setup shown in Fig. 2(b), resulting in the image as shown in the zoomed view in Fig. 3(a).

\section{Industrial Pilot Mill}

An interstitial free steel strip is processed inside a largescale pilot mill with $393.4 \mathrm{~mm}$ diameter and $360 \mathrm{~mm}$ wide work rolls, at a Tata Steel (IJmuiden, The Netherlands), as shown in Fig. 3(b). The material can be cold rolled with varying entry and exit strip tension, velocity, friction or rolling force. Two continuous LED light sources (Scangrip Uniform 03.5407) located on both sides of the camera are pointed towards the steel strip, as can be seen in Fig. 3(b), which also shows an example of the obtained images. Due to limited accessibility, the camera and lights could only be placed outside the work rolls between the bearing chocks of the work rolls, therefore, significant effort was put in optimizing the illumination and camera settings. The images are captured using a Basler scout scA1400-30gm camera. This camera has a resolution of $1392 \times 1040$ pixels, a working distance of $130 \mathrm{~mm}$ due to an additional lens (EDMUND Optics Techspec 50mm/ $F 2.059873$ ) and a maximum frame rate of $25 \mathrm{fps}$, which was also the frame rate used in all pilot mill experiments. The steel strip has a thickness and width of, respectively, $2.96 \mathrm{~mm}$ and $100 \mathrm{~mm}$ and, again, a speckle pattern was created using graphite spray paint. The large exerted vertical force between the rolls deforms the steel strip, by means of cross-expansion, in out-of-plane direction, which results in a change of reflection in horizontal directions, therefore, reflection cannot be avoided in the images, as can be seen in the zoomed view in Fig. 3(b). Normally, during strip rolling, the strip is located in the center line of the rolls, however, because of the low working distance of the cameras, the strip is placed off-centric such that the monitored strip edge is located $30 \mathrm{~mm}$ away from the work rolls' edges. The strips are processed using four different work roll forces $(500,750,1000$ and $1250 \mathrm{kN})$, three different entry strip tensions $(10,15,20 \mathrm{kN})$, and four different exit strip tensions $(10,15,20,30 \mathrm{kN})$, while the processing velocity is fixed at $10 \mathrm{~mm} / \mathrm{s}$, which is much larger than regular DIC experiments. Therefore, the exposure time of the camera becomes important. It has been optimized to a short exposure time of $0.6 \mathrm{~ms}$, because a higher exposure time results in blurred images, whereas for an even shorter exposure time insufficient light is captured for the camera-lighting setup used. With this choice, the image blur remains limited to 0.6 pixel. For (much) higher strip speeds, one should realize that the accuracy of DIC in general start to significantly deteriorate from an image blur of three pixels [34]. The method validation was performed using the images obtained for the $500 \mathrm{kN}$ work roll force and an entry and exit strip tension of 10 and $20 \mathrm{kN}$.

It can directly be seen that the quality of the images obtained from the hand mill setup is significantly better than that obtained from the pilot mill setup due to the significantly larger exposure time and less restricted view. In addition, the deformation zone is longer for the pilot mill, Therefore, the number of pixels over the thickness of the steel strip is lower compared to the hand mill (1392 pixels vs 2592 pixels).

\section{Method Optimization and Validation}

First, an analysis is conducted to select the optimum kinematic regularization that is rich enough to accurately describe the displacement field between the work rolls, but with as few as possible DOFs for maximum correlation robustness. Subsequently, the robustness of the method is tested by omitting a random image in the deformation sequence, thus requiring the algorithm to automatically find a twice as large displacement field by doubling the value for the velocity corrector, after which the uncertainty in the velocity corrector is analyzed. Finally, the absolute deviation of the horizontal and vertical displacement fields for increasing number of images is demonstrated.

\section{Optimization of the Kinematic Regularization}

A polynomial basis for the kinematic regularization is chosen over for example a B-spline grid, because for a reasonably high polynomial order, the kinematic freedom should be sufficiently rich to properly capture the global deformation phenomena that are expected to occur in the 
deformation zone. As visible in Fig. 3, the ROI is a long thin strip, therefore, from a mechanics point of view, it is obvious that the kinematic regularization does not need to be equally rich in thickness and length direction. In thickness direction, a $1^{\text {st }}$ order polynomial in $y$ (with much higher order in $x$ ) would capture the major deformation modes, i.e. compression that is uniform over the thickness but varying along the strip length $\left(\lambda_{\epsilon_{y y}}(x) y \epsilon_{y}\right)$, cross-expansion along the length that is uniform over the thickness $\left(\lambda_{\epsilon_{x x}}(x) \mathbf{\epsilon}_{x}\right)$, and out-of-strip-plane bending around the work rolls $\left(\lambda_{\epsilon_{b}}(x) y \mathbf{\epsilon}_{x}\right)$, where the dependence of the DOFs on $x$ (but not on $y$ ) shows that the magnitude of these deformation modes change along the strip length. This can also describe other possible minor deformation modes, such as rotation of the strip edge towards the camera. Yet, the in-plane shear strain may perhaps be larger close to the work rolls compared to the center line of the sheet, which would require (if present) a $2^{\text {nd }}$ order polynomial description in $y$; this would also capture out-of-image-plane bulging of the strip edge. Therefore, even though the shear strains are small, as will be shown below, in this work a $3^{\text {rd }}$ order polynomial in thickness direction is selected to be on the safe side. This also allows to capture unknown effects due to possible asymmetric feed of the strip into the work rolls. On the other hand, in the length direction of the strip a much higher order regularization is required since the pressure underneath the work rolls varies strongly along the strip length. A higher order is required particularly for the hand mill because the rubber will elastically spring back to its "initial" thickness. Yet, the optimal polynomial order in length direction is not known a priori, therefore the effect of the polynomial order in $\mathrm{x}$-direction is investigated, similar to what was done in $[22,25]$. To this end, polynomials with order 4 to 12 and order 3 in, respectively, strip length and thickness direction were used to correlate the obtained images for both mills (60 images for the hand mill and 200 images for the pilot mill). Each correlation yields a full $\epsilon_{x x}^{\text {tot }}, \epsilon_{y y}^{\text {tot }}$ and $\epsilon_{x y}^{\text {tot }}$ strain field (and a full $v_{x}$ and $v_{y}$ velocity field), as explained in Subsection 2.3 and which will be shown in Section 5. Note that, because it is known that a polynomial description tends to be less accurate at the borders of the field of view, as analysed in [22], a narrow border of ten pixels is removed from the FOV for all presented data.

To assess the effect of the polynomial order on the strain fields, the thickness-averaged total strain profile $\left(\bar{\epsilon}_{x x}^{t o t}, \bar{\epsilon}_{x y}^{\text {tot }}\right.$, and $\left.\bar{\epsilon}_{y y}^{\text {tot }}\right)$ over the length of the strip for both mills are presented in Fig. 4.

Of course, the true strain field is not known, yet careful analysis of the strain fields shows that, for both mills, a minimal polynomials order of 8 in length direction should suffice, since the solution saturates for the $8^{\text {th }}, 9^{\text {th }}$, and $10^{\text {th }}$ polynomial order showing an absolute difference in strain that is (much) less than $1 \%$, for all strain components, anywhere along the strip. Interestingly, the solution starts to significantly deviate again for an order above 10 , showing physical phenomena that are highly unexpected, as can be seen, e.g., for the thickness-averaged total shear strain $\left(\bar{\epsilon}_{x y}^{\text {tot }}\right)$ of the hand mill correlated using an $11^{\text {th }}$ or $12^{\text {th }}$ order polynomial. Moreover, the magnitude and frequency of such unexpected, nonphysical fluctuations further increases for even higher polynomial degrees (not shown). This demonstrates the importance of selecting a rich, but not too rich kinematic description to maximize correlation robustness. Therefore, a polynomial with $8^{\text {th }}$ order in length direction seems to be the optimal choice, and is used for all the following correlations. Hence, for both mills, a polynomial with, respectively, $8^{\text {th }}$ and $3^{\text {rd }}$ order in length and thickness direction is chosen, resulting in 60 spatial DOFs. Furthermore, the (field averaged) residual has been studied as a function of the polynomial order and shows, as expected, a continuous decrease up to $8^{\text {th }}$ order, after which it stagnates until the $10^{\text {th }}$ order. From $11^{\text {th }}$ order and higher, the residual starts to increase slightly, while the number of iterations to find the optimal solution increases drastically. Therefore, this trend in the (field averaged) residual provides additional support to the conclusion that an $8^{\text {th }}$ order polynomial in strip length direction appears to be optimal. Note that in a future publication [35] (where the here-proposed methodology is used as an established method to resolve some of the intricate details of the cold strip rolling process) a $4^{\text {th }}$ order polynomial in strip length direction is used because in that work pre-rolled steel strips are tested, resulting in nearly perfect horizontal material feed and hence a more homogeneous and symmetric strain field, thus requiring a lower kinematic description. In general, please note that the choice of the basis is not a critical part of the proposed methodology, and a preliminairy test (not shown) indicates that a set of harmonic basic functions could also be made to work. However, a set of smooth global basis functions should be selected, because even a grid of (rather smooth) $6^{\text {th }}$-order B-spline basis functions introduced insufficient kinematic regularization for the challenging case of image correlation of strip rolling.

\section{Robustness Analysis}

With the order of the polynomial kinematic basis optimized, the precision and robustness with which the velocity corrector, $\alpha_{t}$, can be identified is unknown. Therefore, additional correlations are performed in which, respectively, the 60 and 200 images are again correlated for the hand mill and pilot mill case, however, for both cases the fifth image has been removed. Removing one of the images results in an approximately twice as large displacement difference for the image pair containing image four and six. Since the 


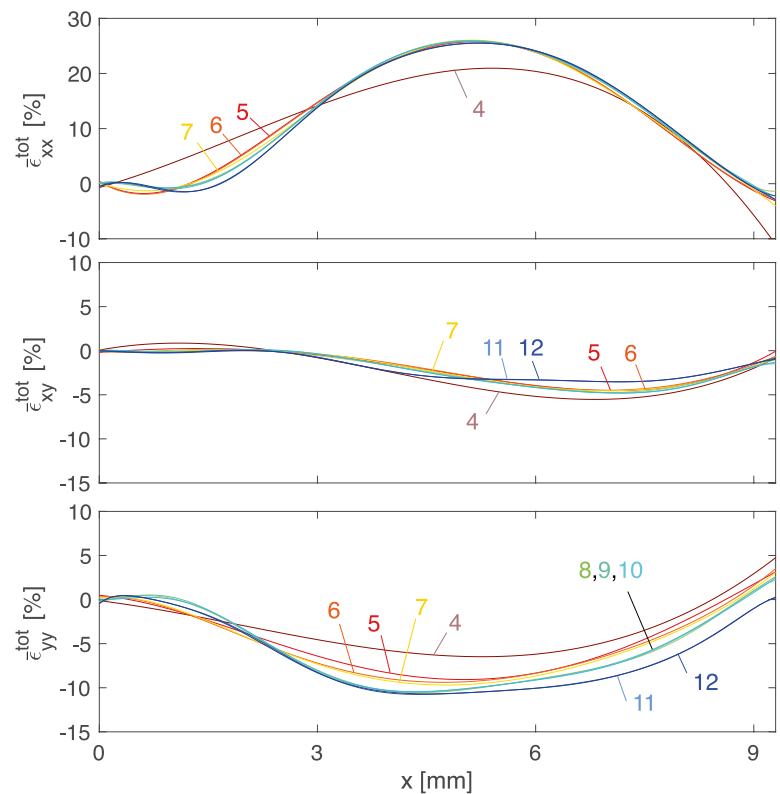

(a) - Hand mill

Fig. 4 Thickness averaged total strain profiles $\left(\bar{\epsilon}_{x x}^{\text {tot }}, \bar{\epsilon}_{x y}^{\text {tot }}\right.$, and $\left.\bar{\epsilon}_{y y}^{\text {tot }}\right)$ over the length of the strip of, respectively, the hand mill and pilot mill for polynomials with orders 4 to 12 in length and order 3 in thickness direction. The solution has saturated after a $8^{\text {th }}$ order polynomial

velocity corrector is multiplied with the average displacement field, the value of factor $\alpha_{t}^{4-6}$ between image pair four and six should be equal to the value of $\alpha_{t}^{4-5}$ plus $\alpha_{t}^{5-6}$, i.e. the value of $\alpha_{t}^{4-6}$ in the solution should be twice that of its initial guess. It was found that, for both the hand and pilot mill image series, the solution algorithm has no problem at all in finding this global minimum starting from a distant initial guess, demonstrating the robustness of the t-IDIC framework.

It is also interesting to investigate the accuracy of the final displacement field with the fifth image omitted for the correlation. The results from both mills are shown in Table 2, resulting in a difference of, respectively, $0.25 \%$ and $0.28 \%$ with or without the fifth image. Please note that the difference between $\alpha_{t}^{4-5}$ and $\alpha_{t}^{5-6}$ for the different image pairs of the hand mill is larger than the pilot mill because of the large variability due to manual operation.

It must be taken into account that removing one image changes the average deformation field as well, hence a small deviation will be present for all the other $\alpha_{t}$ factors. The mean absolute difference of all $\alpha_{t}$ values for the two correlations when the $5^{\text {th }}$ image is taken out equals, $0.09 \%$ and $0.004 \%$ for, respectively, the hand and pilot mill. These small values imply that the above-mentioned difference between $\alpha_{t}^{4-5}+\alpha_{t}^{5-6}$ and $\alpha_{t}^{4-6}$ corresponds to an error level similar to if there would only be image noise, although other

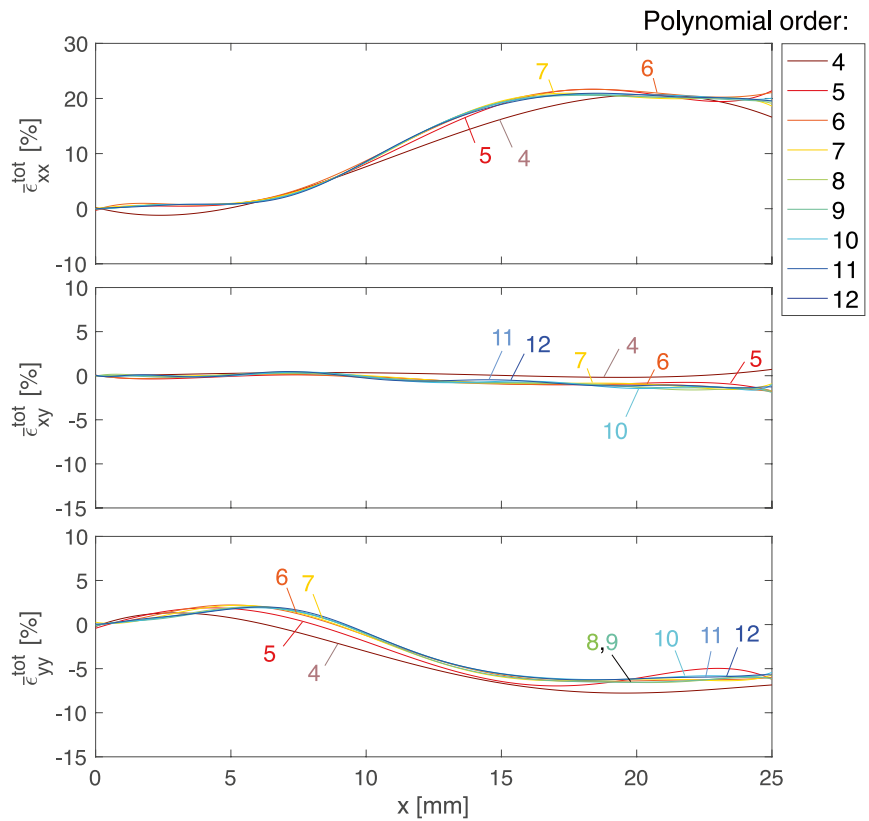

(b) - Pilot mill

in length direction, therefore, an $8^{\text {th }}$ order polynomial is used in this work. Note that choosing a too large order (i.e. 11 or 12) results in deviations from the solution

Table 2 Results from correlating 60 and 200 images for respectively, the hand and pilot mill, using (i) all images are used to find convergence and (ii) the $5^{\text {th }}$ image is taken out and convergence is again found. A difference of $0.25 \%$ and $0.28 \%$ is found for, respectively, the hand mill and pilot mill, when comparing $\alpha_{t}^{4-5}+\alpha_{t}^{5-6}$ versus $\alpha_{t}^{4-6}$, where it is noted that it remains unknown which of the two is more accurate. The mean absolute difference found in all the values of $\alpha_{t}$ for the hand and pilot mill when the $5^{\text {th }}$ image is taken out equals, respectively, $0.09 \%$ and $0.004 \%$, which are of negligible magnitude

\begin{tabular}{cc}
\hline Hand Mill & 60 images \\
\hline$\alpha^{4-5}$ & $=0.767$ \\
$\alpha^{5-6}$ & $=0.821$ \\
$\alpha^{4-5}+\alpha^{5-6}$ & $=1.588$ \\
\hline $5^{\text {th }}$ image removed & 59 images \\
\hline$\alpha^{4-6}$ & $=1.585$ \\
\hline Difference $\alpha^{4-5}+\alpha^{5-6}$ and $\alpha^{4-6}$ & $=0.25 \%$ \\
\hline Mean difference on all $\alpha_{t}$ values & $=0.09 \%$ \\
\hline Pilot Mill & $\mathbf{2 0 0}$ images \\
\hline$\alpha^{4-5}$ & $=0.959$ \\
$\alpha^{5-6}$ & $=0.959$ \\
$\alpha^{4-5}+\alpha^{5-6}$ & $=1.918$ \\
\hline $5^{\text {th }}$ image removed & 199 images \\
\hline$\alpha^{4-6}$ & $=1.923$ \\
\hline Difference $\alpha^{4-5}+\alpha^{5-6}$ and $\alpha^{4-6}$ & $=0.28 \%$ \\
\hline Mean difference on all $\alpha_{t}$ values & $=0.004 \%$
\end{tabular}




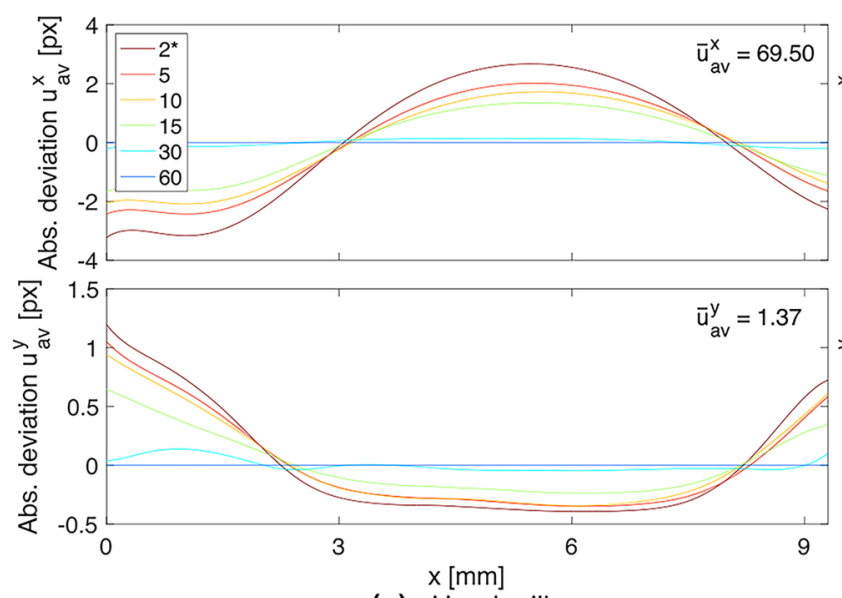

(a) - Hand mill

Fig. 5 The thickness-averaged absolute average displacement $\left(u_{a v}\right)$ deviation in $\mathrm{x}$ - and $\mathrm{y}$-direction along the strip, where the deviation is assumed zero for the correlation which used the maximum number of images, with (a) the hand mill and (b) the pilot mill for increasing amount of images used. The solution found after correlating the

minor error sources may still exist. Note that the effect on the finally identified displacement field, i.e. $\bar{\alpha}_{t} \underline{u} a v$, will be much smaller as the uncertainty on $\bar{\alpha}_{t}$ averages out with the square root of the amount of images are considered (e.g. for 200 images, the uncertainty in the displacement field will be $0.004 \% / \sqrt{200} \approx 0.0003 \%$ ). The average deviation of $0.09 \%$ for the hand mill will most likely come closer to zero if an increased number of images were correlated, as was done for the pilot mill.

\section{Accuracy Analysis}

To further investigate the accuracy and robustness of the proposed t-IDIC method, the images obtained from both test cases are correlated with increasing number of images. For the hand mill, a total of 60 images were considered, while 200 images were considered for the pilot mill because of the lower image quality. For both cases, the kinematic regularization proposed in Subsection 4.1 is used to correlate the obtained images. The thickness-averaged absolute average displacement $\left(u_{a v}\right)$ deviation in $\mathrm{x}$ - and $\mathrm{y}$-direction along the length of the strip for both mills are given in Fig. 5, where the reference trends (deviation of zero) were taken as the correlation with the maximum amount of images. Figure 5 shows, for both mills, a converging trend towards a high accuracy solution when more images are used in the correlation. For the hand mill and pilot mill the solution converges after, respectively, using 30 images and 100 images, hence a highly accurate solution is found when using, respectively, 60 and 200 images. Note that the order of magnitude difference in the deviation between the hand and pilot mill can be attributed to (i) the increased pattern quality, which was

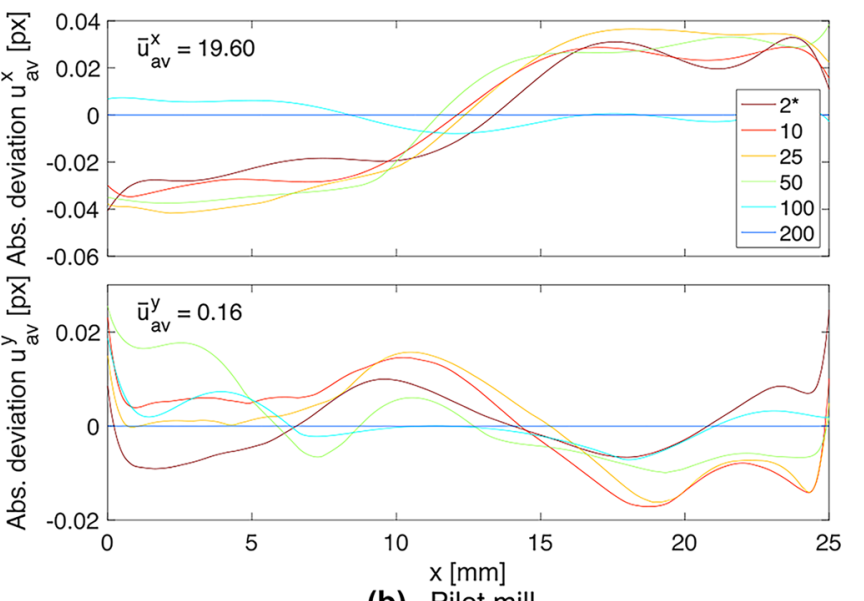

(b) - Pilot mill

maximum number of images is chosen as a reference. A converging trend of the solution is visible for both mills, clearly showing that an increased amount of images results in a lower deviation and hence higher accuracy. *Note that using only two images corresponds to regular GDIC

meticulously optimized, and (ii) the much better camera and lens setup used during the pilot mill case (resulting in an increased DIC accuracy), see Fig. 3. Finally, Fig. 5(b) also shows that the displacement accuracy for the pilot mill case when correlating a 200 images series appears to be below 0.01 pixel, which can be considered as very good, considering the highly challenging imaging conditions, even though the spatial resolution of the strain field is (on purpose) limited.

\section{Proof-of-Principle Demonstrations}

First the richness of the full-field results generated by the proposed t-IDIC methodology, i.e. the obtained residual, velocity fields, strain fields and surface area ratio field is demonstrated for the hand mill and pilot mill. Then two specific test cases are elaborated for the pilot mill by (i) varying the work roll pressure and (ii) the entry and exit strip tension. At last, an extension of the novel method is proposed in which two parallel FOVs of the deformation zone are correlated simultaneously, to further increase the accuracy of the correlation's outcome.

\section{Demonstration of the Richness of the Full-Field Results}

\section{Hand Mill}

The results from correlating 60 deformed images obtained using the hand mill are given in Fig. 6, with (a) showing the first of 60 images. The averaged residual field $(r)$ for all 


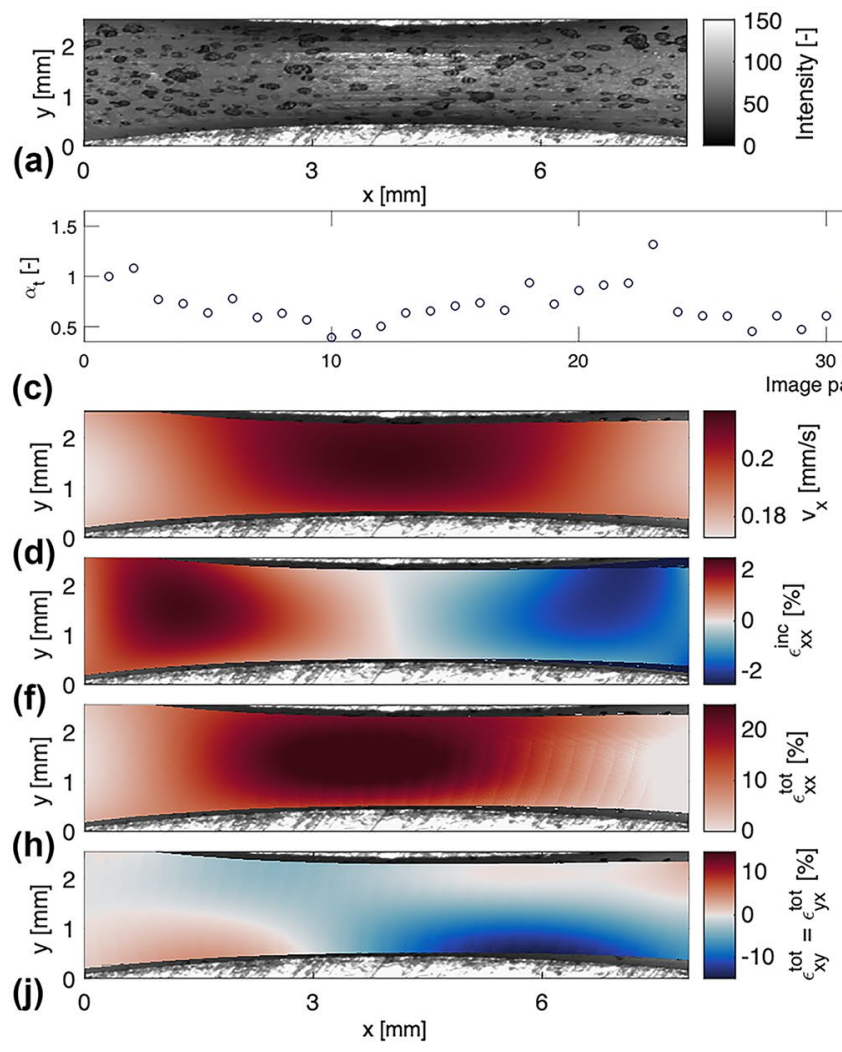

Fig. 6 Results for the hand mill case processing a rubber strip with (a) the first of 60 images, (b) the residual $(r)$, (c) the velocity corrector $\left(\alpha_{t}\right)$ for every image pair, (d) the average horizontal velocity $\left(v_{x}=\frac{\left.\alpha_{t} \underline{\underline{u}_{a v}} \underline{\underline{x}}\right) \cdot \underline{\underline{e}}_{x}}{\delta t}\right)$ with the time between two images being $\delta t \approx 1 \mathrm{~s},(\mathbf{e})$ the average vertical velocity $\left(v_{y}\right)$, (f) the incremental horizontal strain

the correlated images is given in Fig. 6(b), where a pattern of low and high residual is found between $2<x<6 \mathrm{~mm}$, which remains visible for higher order polynomials (even up to $12^{\text {th }}$ order), hence it can be concluded that this pattern results from unavoidable light reflections. Note that all images could be combined to create a "background image" in which the speckle pattern has dissapeared due to averaging, thus only showing the consistently overexposed regions caused by reflections, while subtracting this background image results in background-corrected images from which the reflections have been mostly removed (but not completely as the reflections vary between images). However, using these background-corrected images does not at all improve the quality of the DIC correlation or the accuracy of the strain and velocity fields (as is logical from a theoretical point of view as background subtraction results in the subtraction of the same quantity on both sides of equation (3), thus having no effect on the correlation), whereas it does make the residual field more difficult to interpret. Therefore, the uncorrected images are used in this work.

Most excitingly, while the roll velocity of the hand-driven mill is fluctuating heavily, shown by the velocity corrector

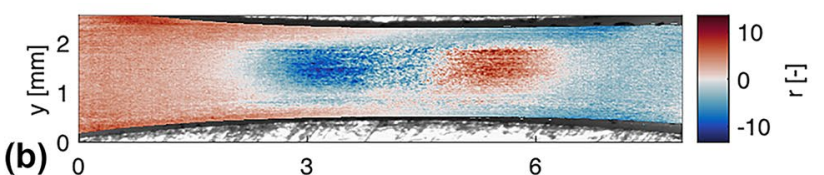

(b) $\begin{array}{lll}0 & & \\ 0 & 3 & \times[\mathrm{mm}]\end{array}$

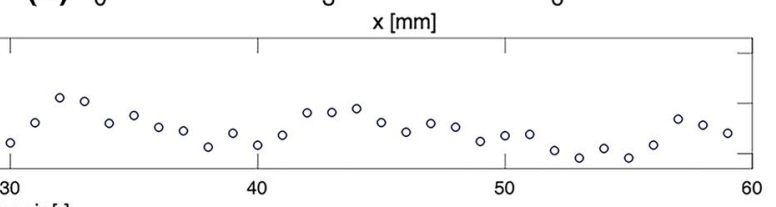

pair [-]

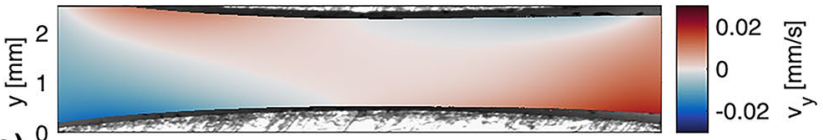

(e)

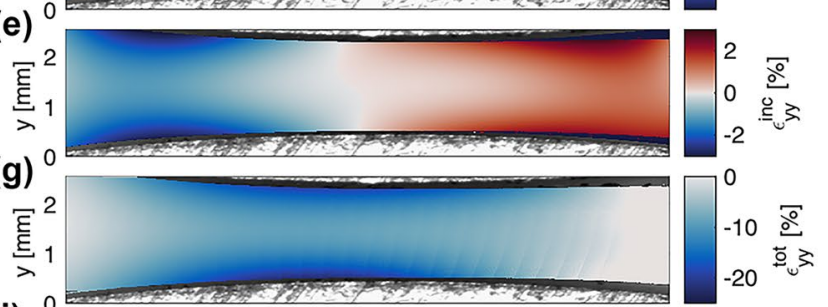

(i)

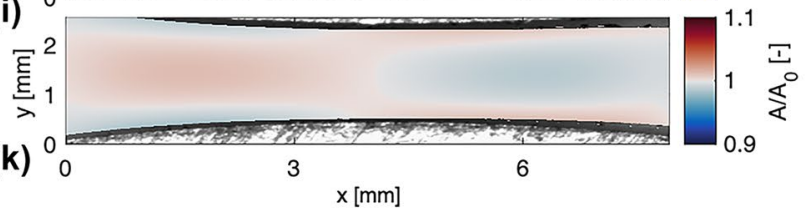

$\left(\epsilon_{x x}^{i n c}\right),(\mathbf{g})$ the incremental vertical strain $\left(\epsilon_{y y}^{i n c}\right),(\mathbf{h})$ the total horizontal strain $\left(\epsilon_{x x}^{t o t}\right),(\mathbf{i})$ the total vertical strain $\left(\epsilon_{y y}^{t o t}\right),(\mathbf{j})$ the total shear strain $\left(\epsilon_{x y}^{t o t}=\epsilon_{y x}^{t o t}\right)$, and $(\mathbf{k})$ the surface area ratio $A / A_{0}$ (i.e. determinant of the total $2 \mathrm{D}$ deformation gradient tensor $\underline{F}_{\text {tot }}^{2 D}$ )

$\left(\alpha_{t}\right)$ for every image pair in Fig. 6(c), the method is still robust enough to properly capture the strip's kinematics. The $\alpha_{t}$ factor fluctuates between 0.5 and 1.5 , where an $\alpha_{t}$ of 0.5 practically means that the average velocity for this image pair is 0.5 times the average velocity of the first image pair (which by default is assigned a value of $\alpha_{t}=1$ ).

The average horizontal and vertical velocity fields given in Fig. 6(d-e) show that after the mill, the material recovers to its initial configuration (the horizontal velocity before and after the work rolls are equal). However, this doesn't happen in a symmetric manner because the material, before and after rolling, is not kept horizontally by means of any strip tension, see Fig. 6(e), where applying a entry and exit strip tension is normal practice in strip rolling. It can be seen that the strip velocity over the thickness is not constant, which is attributed to the strip not being under tension, allowing the top half to deform more than the lower half. Processing rubber strips is a special case, because of its (an-) elastic behavior and spring back after the work roll, which is why there are two neutral points where the strip velocity equals the work roll velocity (upstream and downstream), see Fig. 6(d). Note, however, that the work roll velocity is not known for 
the hand mill, therefore the precise position of the neutral points cannot be displayed in Fig. 6.

The incremental horizontal and vertical strain given in Fig. 6(f-g), very prominently show the potential of the proposed method. Both the horizontal and vertical incremental strain are almost symmetrical in $x$, which can be expected for (an-) elastic material, even though the strains are quite small. This directly shows that the accuracy of the strain components lies far below $1 \%$. Furthermore, the elastic recovery after the strip is released from the work rolls is clearly visible.

The total horizontal and vertical strain fields can be obtained since the displacement fields are known during the whole process (as explained in Subsection 2.3) and are given in Fig. 6(h-i). The $\epsilon_{x x}^{\text {tot }}$ field shows, as expected, an increase when the material is pushed through the mill, reaching a maximum strain of $\sim 25 \%$ after which the strip return to its initial state (i.e. $\epsilon_{x x}^{t o t} \approx 0$ ). The fact that $\epsilon_{x x}^{t o t}$ goes indeed back to zero after the rolls shows the accuracy of the method. Also interesting to observe is that the vertical strain is maximum at the contact where the pressure is applied and decreases towards the center where the material is able to "escape" in horizontal direction, therefore reducing the vertical strain. In contrast, the horizontal strain has its peak at the center line because the horizontal displacement is constrained at the top and bottom of the strip due to friction with the work rolls. The $\epsilon_{y y}^{\text {tot }}$ field shows that the downward bending, due to gravity, of the material has a significant influence on the obtained field, which shows that the methodology is sensitive to strip tension. The total shear strain $\left(\epsilon_{x y}^{\text {tot }}\right.$ or $\left.\epsilon_{y x}^{t o t}\right)$ in Fig. 6(j) shows a negative shear magnitude in the bottom of the material after the mill, which is attributed to the fact that the material is hanging due to gravity.

The surface area ratio $A / A_{0}$ (determinant of the total $2 \mathrm{D}$ deformation gradient tensor $\underline{F}_{t o t}^{2 D}$ ) is given in Fig. 6(k). Even though the magnitude of $\epsilon_{x x}^{\text {tot }}$, $\epsilon_{x x}^{\text {tot }}$ and $\epsilon_{x x}^{\text {tot }}$ are large, $A / A_{0}$ remains very close to 1 , as it should, which is a further confirmation that the method is reliable and accurate and yields physically correct fields. The minor fluctuations from 1 are attributed to the (an-)elastic out-of-plane deformation which of course cannot be captured with this 2D method, as explained in Subsection 2.3.

To conclude, for this hand mill, which lacks standard sensors to measure i.e. roll pressure, these results nicely demonstrate that the richness of the full-field data provides clear insights in the deformation process that far exceeds that of standard sensor readings. The non-ideal processing conditions in the experiment are even useful to show how complicated the (elastic) strip rolling process can be and how well the proposed t-IDIC method performs under such non-ideal conditions.

\section{Industrial Pilot Mill}

The results from correlating 200 deformed images obtained using the industrial pilot mill with a work roll force of 500 $\mathrm{kN}$ and an exit and entry strip tension of, respectively, 10 and $20 \mathrm{kN}$ are given in Fig. 7, with (a) the first image, in which the entry and exit point have been identified using image processing, which is explained in a future publication [35]. The averaged residual field $(r)$ is given in Fig. 7(b) and shows a region of high residual caused by unavoidable light reflections, which can be corrected for using the aboveproposed background correction method, but is not implemented here.

The velocity corrector $\left(\alpha_{t}\right)$ of every simultaneously correlated image pair is given in Fig. $7 \mathrm{c}$ ), showing fluctuations between 0.95 and 1.05 , thus the pilot mills test case is clearly more constant than the hand mill test case. Although these fluctuations are smaller than the hand mill case, they are by no means insignificant and mainly result from camera timing and work roll velocity variations. Interestingly, four image pairs (out of 200) found velocity corrector values which were far outside the $0.95<\alpha_{t}<1.05$ bounds due to a locally poor pattern quality, and were automatically removed from the correlation (indicated by the red crosses at value $\alpha_{t}=1$.) The method is robust enough to still obtain a proper solution for all other images, due to the strong kinematic regularization (the same lower order displacement field for all images) that is at the heart of the correlation framework.

Figure 7(d-e) presents the average velocity fields of the processed steel strip. In Fig. 7(a, d, e), the neutral point has been identified and indicated. These velocity fields clearly show the compression that happens in between the work rolls. Interestingly, Fig. 7(e) shows that the strip first deforms on the top side because it is slightly pre-bent upwards resulting from the fact that the strip is stored on coils which is subsequently guided over guiding rolls where plastic bending occurs. Hence the material is first pushed down by the top work roll until it touches the bottom roll, which is in good agreement with the different locations of the entry points of the top and bottom work roll, see Fig. 7(e). Please note that even though the strip is subjected to a relatively high entry and exit strip tension, the material is still fed under an angle, which was unexpected and has been very well visualized by the novel proposed t-IDIC method. Additionally, the diameters of the used work rolls are 393.36 and 393.38 $\mathrm{mm}$, hence this minor difference could also not explain the non-horizontal material feed.

The incremental horizontal and vertical strain are given in Fig. 7(f-g), in which the asymmetry due to a non-horizontal material feed is clearly visible (especially in Fig. 7(g)). Both incremental strain fields show that the main compression 


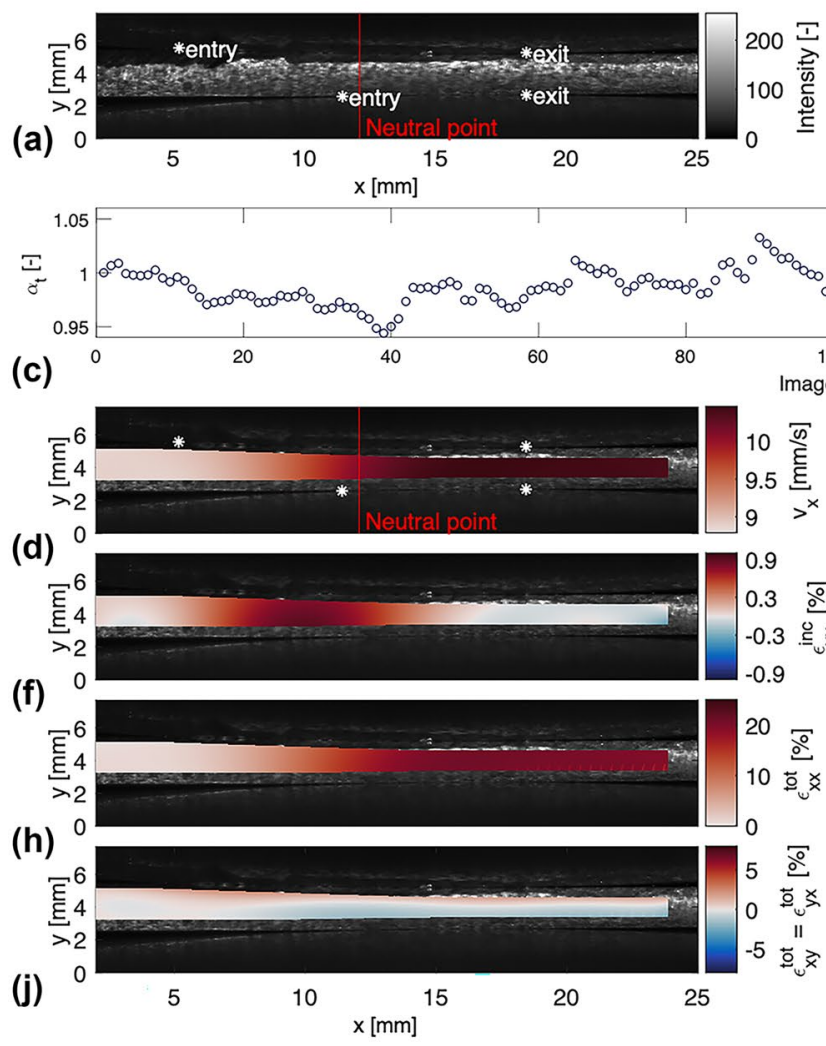

Fig. 7 Results for the pilot mill case, processing a steel strip with a work roll force of $500 \mathrm{kN}$ and an exit and entry strip tension of, respectively, 10 and $20 \mathrm{kN}$, with (a) the first image with the entry and exit points given, (b) the residual $(r),(\mathbf{c})$ the velocity corrector $\left(\alpha_{t}\right)$ for every image pair, (d) the average horizontal velocity

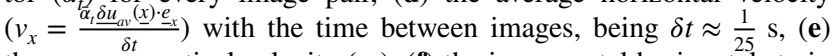
the average vertical velocity $\left(v_{y}\right),(\mathbf{f})$ the incremental horizontal strain

part occurs in between both entry points, even though the strains are low, providing direct evidence of the method accuracy. To further show the method capabilities applied to an industrial pilot mill, the (positive) elastic recovery (which is known to be small) of the strip after the exit point is clearly visible in Fig. 7(g).

The horizontal and vertical total strain fields are shown in Fig. 7(h-i). These strain fields clearly show vertical compression of the strip during rolling, resulting in a significant decrease in $\epsilon_{y y}^{\text {tot }}$ below 0 and an almost equally large increase in $\epsilon_{x x}^{\text {tot }}$, corresponding to a strain state of the image side surface of the strip that is nearly plane strain, as expected. The slightly larger magnitude of $\epsilon_{y y}^{t o t}$ on the top side of the strip, just after the top entry point, is attributed to the off-centric alignment due to non-horizontal material feed. In [35], the here-proposed method is employed on a large set of (different) pilot mill experiments, where it will be shown that the asymmetry can be remedied by prerolling the strip to minimize the bend (without passing the $\left(\epsilon_{x r}^{i n c}\right),(\mathbf{g})$ the incremental vertical strain $\left(\epsilon_{y y}^{i n c}\right),(\mathbf{h})$ the total horizontal strain $\left(\epsilon_{x x}^{t o t}\right)$, (i) the total vertical strain $\left(\epsilon_{y y}^{t o t}\right),(\mathbf{j})$ the total shear strain $\left(\epsilon_{x y}^{t o t}=\epsilon_{y x}^{t o t}\right)$, and $(\mathbf{k})$ the surface area ratio $A / A_{0}$ (determinant of the deformation total $2 \mathrm{D}$ gradient tensor $F_{\text {tot }}^{2 D}$ ) with the red crosses indicating falsely converged images due too poor image quality or pattern degradation which are removed from the total correlation. The entry, exit and neutral points are also added

strip and guiding roll), which confirms the validity of the current measurements and statements. In Subsections 5.2 and 5.3 below, the work roll force and the entry strip tension is increased, which results in a more horizontal material feed and hence more symmetrical strain fields, providing further confirmation. In Fig. 7(j), the shear strain $\left(\epsilon_{x y}^{\text {tot }}\right.$ $\left.=\epsilon_{y x}^{t o t}\right)$ field shows that positive and negative values are found for, respectively, the top and bottom half of the strip and approximately zero in the center. The found shear field is as expected, while the rolls pull the strip through the mill having almost symmetric shear with a zero line at the center of the strip.

Figure $7(\mathrm{k})$ shows the surface area ratio $A / A_{0}\left(\operatorname{det}\left(\underline{F}^{2 D}\right)\right)$ which remains close to 1 , resulting in a value just below 1 downstream due to out-of-plane deformations which are not captured by the proposed t-IDIC method. This expected behavior from a mechanics point of view is further evidence that the method is reliable, robust and accurate enough to properly capture the strip's kinematics. 
The proposed t-IDIC methodology allows comparison of all the rich data fields to study the strip roll process in detail. For instance, investigation of the velocity and strain fields with respect to the position of the entry/ exit point and neutral point can show pre-deformation before the material enters the work rolls. Combining this with the observed strip velocity gradient over the thickness, it is clear that the proposed t-IDIC methodology provides valuable novel insights of processes occurring in the deformation zone, as is exploited, e.g., in [35].

\section{Case Study I: Variation of the Work Roll Force}

It has been demonstrated that the proposed t-IDIC method allows identification of the most significant deformation fields and rolling parameters of a strip rolling process. The potential of the methodology is further demonstrated on two case studies. In the first case study (CS I), the work roll force is varied $(500,750,1000,1250 \mathrm{kN})$, while keeping the strip tension constant at $10 \mathrm{kN}$ and $20 \mathrm{kN}$ for, respectively, the entry and exit strip tension and the rolling velocity is kept at $10 \mathrm{~mm} / \mathrm{s}$. All used and obtained processing conditions and parameters are given in Table 3 , in which the entry $\left(v_{i n}\right)$, the work roll $\left(v_{\text {roll }}\right)$, and the exit velocity $\left(v_{\text {exit }}\right)$, each logged using an in-built velocity sensors are given. The exit thickness is determined using (i) the mass flow equation, i.e. $v_{\text {in }} t_{\text {in }}=v_{\text {out }} t_{\text {out }}\left(t_{M F}\right)$ and (ii) the average vertical strain after the exit point $\left(t_{D I C}\right)$ and applied to the initial strip thickness $(2.96 \mathrm{~mm})$. Table 3 also shows the surface area ratio with standard deviation considering all data points after the exit point $\left(A / A_{0}\right)$, and the horizontal position of the neutral point with respect to the exit point position $\left(x_{n p}\right)$.

The difference between $t_{M F}$ and $t_{D I C}$ can be explained by the possibility that the strip already deforms before the FOV, implying that capturing the strip before the deformation zone may be significant, as is done in Section 5.4. Table 3 shows that, as expected, a larger work roll force results in a larger reduction in exit thickness, a reduction in $v_{i n}$, and the neutral and exit point shift closer to each other, all of which show an expected monotonic trend, confirming the reliability of the method. Furthermore, the surface area ratio after the exit point is just below 1 for each work roll force, further supporting the reliability of the obtained data. The full-field results for the different work roll forces are given in Fig. 8. An increased work roll force results in a larger thickness reduction (see Table 3), which subsequently results in a larger contact area and lower entry velocity and slightly higher exit velocity, as can be seen in the horizontal velocity $\left(v_{x}\right)$ fields presented in Fig. 8(a).

For each work roll force, the entry and exit points and neutral point are computed and shown. An increasing work roll force results in a larger distance between the neutral point and the entry point, which is expected because the larger thickness reduction, a larger torque must be transmitted through the interface, leading to an increased backward slip zone. Furthermore, the vertical velocity $\left(v_{y}\right)$ fields show a larger negative velocity after the first entry point for an increased work roll force, which results in a more horizontal material feed. This can also be seen in the strains fields given in Fig. 8(c-e). The gradient in the low total vertical strain $\left(\epsilon_{y y}^{\text {tot }}\right)$ over the thickness of the strip, which is caused by the pre-bending prior to rolling disappears for an increasing work roll force, as this pre-bending occurs more outside the FOV and can thus not be taken into account in the total strain calculation. Furthermore, an increase in the magnitude of the total horizontal strain $\left(\epsilon_{x x}^{\text {tot }}\right)$ and total vertical strain $\left(\epsilon_{y y}^{\text {tot }}\right)$ is visible for increasing work roll force as expected. The total shear strain $\left(\epsilon_{x y}^{\text {tot }}\right)$ shows that the process becomes more homogeneous throughout the FOV for increasing work roll force, i.e. the strain gradients reduce.
Table 3 Processing settings along with obtained properties and parameters for case study I (variation of the work roll force) and case study II (variation of the strip tension). The entry $\left(v_{i n}\right)$, work roll $\left(v_{\text {roll }}\right.$ and exit velocity $\left(v_{\text {exit }}\right)$ are measured using built-in velocity sensors. The exit thickness is determined by means of mass flow conservation, i.e. $v_{\text {in }} t_{\text {in }}=v_{\text {out }} t_{\text {out }}\left(t_{M F}\right)$ and by using the average total vertical strain after the exit point to the initial thickness $\left(t_{D I C}\right)$. The average surface area ratio after the exit point $\left(A / A_{0}\right)$, and the horizontal position of the neutral point with respect to the exit point $\left(x_{n p}\right)$ are given. The standard deviation of $A / A_{0}$ is determined by considering all data points to the right of the exit point

\begin{tabular}{llllllllll}
\hline & $F_{\text {entry }}-F_{\text {exit }}[\mathrm{kN}]$ & $F_{\text {roll }}[\mathrm{kN}]$ & $v_{\text {in }}[\mathrm{mm} / \mathrm{s}]$ & $v_{\text {roll }}[\mathrm{mm} / \mathrm{s}]$ & $v_{\text {exit }}[\mathrm{mm} / \mathrm{s}]$ & $t_{M F}[\mathrm{~mm}]$ & $t_{D I C}[\mathrm{~mm}]$ & $A / A_{0}[-]$ & $x_{\text {np }}[\mathrm{mm}]$ \\
\hline CS I & $10-20$ & 500 & 8.875 & 9.93 & 10.367 & 2.534 & 2.535 & $0.9995 \pm 0.0017$ & 5.37 \\
& $10-20$ & 750 & 8.466 & 10.01 & 10.589 & 2.367 & 2.466 & $0.9981 \pm 0.0016$ & 5.27 \\
& $10-20$ & 1000 & 7.940 & 9.89 & 10.500 & 2.238 & 2.396 & $0.9975 \pm 0.0025$ & 5.15 \\
& $10-20$ & 1250 & 7.680 & 9.94 & 10.629 & 2.139 & 2.214 & $0.9970 \pm 0.0032$ & 5.01 \\
CS II & $10-10$ & 750 & 8.458 & 9.97 & 10.449 & 2.396 & 2.561 & $0.9961 \pm 0.0027$ & 6.06 \\
& $10-20$ & 750 & 8.335 & 9.90 & 10.398 & 2.373 & 2.560 & $0.9961 \pm 0.0026$ & 5.92 \\
& $10-30$ & 750 & 8.387 & 9.95 & 10.514 & 2.361 & 2.493 & $0.9958 \pm 0.0030$ & 5.85 \\
& $15-15$ & 750 & 8.340 & 9.86 & 10.363 & 2.382 & 2.565 & $0.9980 \pm 0.0024$ & 6.02 \\
& $20-10$ & 750 & 8.470 & 9.95 & 10.417 & 2.407 & 2.525 & $0.9972 \pm 0.0022$ & 5.89 \\
\hline
\end{tabular}



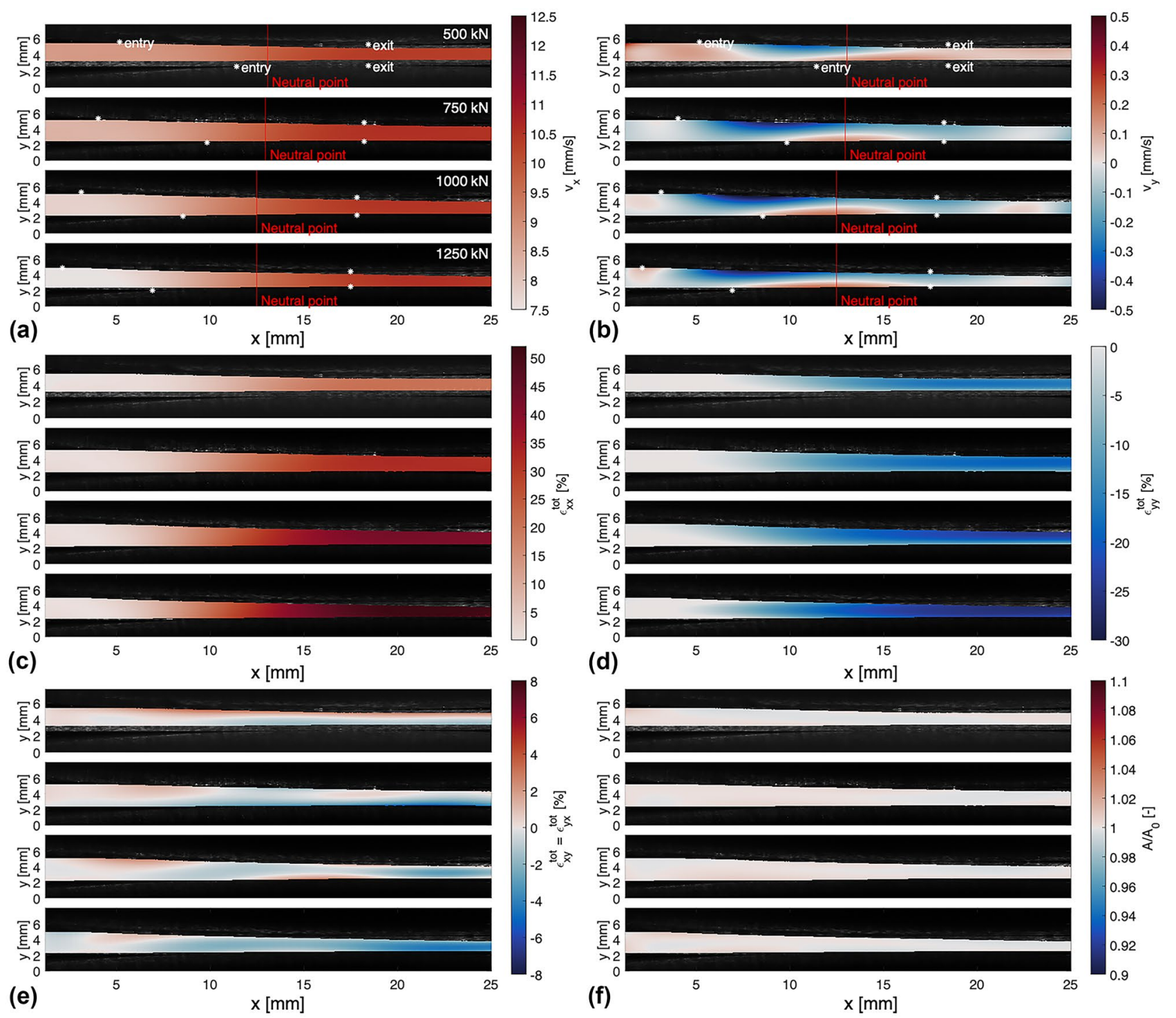

Fig. 8 Results for the pilot mill case using different work roll forces $(500,750,1000,1250 \mathrm{kN})$ and constant strip tension $(10-20 \mathrm{kN})$, with (a) the average horizontal velocity $\left(v_{x}\right)$, (b) the average vertical velocity $\left(v_{y}\right),(\mathbf{c})$ the total horizontal strain $\left(\epsilon_{x x}^{t o t}\right),(\mathbf{d})$ the vertical

strain $\left(\epsilon_{y y}^{\text {tot }}\right),(\mathbf{e})$ the shear strain $\left(\epsilon_{x y}^{\text {tot }}\right)$ and (f) the the surface area ratio $\left(A / A_{0}\right)$. Significant rolling parameters, notably the entry, exit and neutral point, are indicated in (a-b), showing that their location shifts towards the entry for increasing work roll force

Finally, the surface area ratio $\left(A / A_{0}\right)$ is very close to 1 for all tests, clearly showing that the results are reliable.

\section{Case Study II: Variation of the Strip Tension}

In the second case study (CS II), the influence of the entry and exit strip tension is investigated. Controlling the strip tension during cold rolling is important to achieve stable rolling, reduce work roll force and ensure strip quality. The entry and exit strip tension $\left(F_{\text {entry }}\right.$ and $\left.F_{\text {exit }}\right)$ is varied to 10 - $10,10-20,10-30,15-15$ and $20-10 \mathrm{kN}$, while the work roll force and velocity is kept constant at, respectively, $750 \mathrm{kN}$ and $10 \mathrm{~mm} / \mathrm{s}$. All used and obtained processing

conditions and parameters are given in the bottom of Table 3. Table 3 shows that an increase of the exit and entry tension results in, respectively, a decrease and increase of the thickness reduction according to the values of $t_{M F}$ and $t_{D I C}$.

The full-field results for the different strip tension combinations are given in Fig. 9. Increasing the tension at the exit results in a larger strip velocity at the entrance and therefore the neutral point, indicated in Fig. 9(a-b), shifts towards the entry. For increasing entry tension the opposite holds, i.e. the strip velocity decreases and the neutral point shifts towards the exit. When increasing both strip tensions in the fourth test (of CS II), the neutral point stays approximately at the same position compared to the first test. The average 

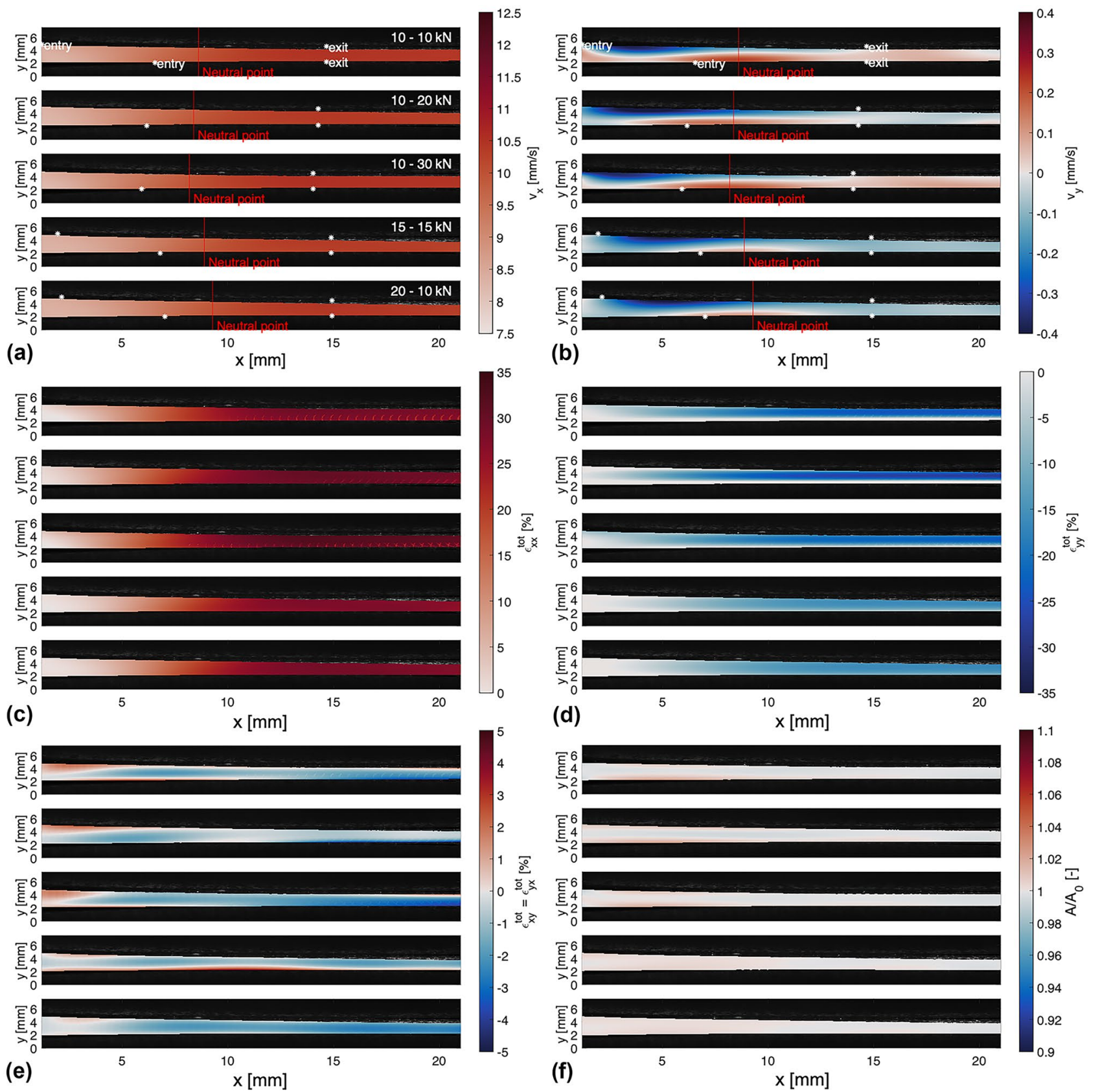

Fig. 9 Results for the pilot mill case using different strip tension combinations $\left(F_{\text {entry }}-F_{\text {exit }}: 10-10,10-20,10-30,15-15,20-10 \mathrm{kN}\right)$, with (a) the average horizontal velocity $\left(v_{x}\right),(\mathbf{b})$ the average vertical velocity $\left(v_{y}\right),(\mathbf{c})$ the total horizontal strain $\left(\epsilon_{x x}^{\text {tot }}\right),(\mathbf{d})$ the total vertical strain $\left(\epsilon_{y y}^{t o t}\right),(\mathbf{e})$ the total shear strain $\left(\epsilon_{x y}^{\text {tot }}\right)$ and (f) surface area ratio $\left(A / A_{0}\right)$. An increase in exit strip tension results in the neutral point

shifting towards the entry, an increase in entry tension shifts the neutral point to the exit. A larger entry strip tension promotes a more horizontal feed of the material, resulting in a more symmetric rolling process. Note that the images in this case study (CS II) were captured approximately $4.5 \mathrm{~mm}$ to the left than CS I

vertical velocity given in Fig. 9(b) can reveal novel insights on the symmetry of the rolling process and the influence of the entry and exit tension. For an entry tension of $10 \mathrm{kN}$, the strip first touches the top work roll, resulting in a slight downwards bending before rolling (as seen previously), this downwards bending was also visible for the tests with increasing exit strip tension. When increasing the entry

tension (to 15 or $20 \mathrm{kN}$ ), a more horizontal material feed is realized, resulting in a decreased vertical velocity gradient, as shown in Fig. 9(b), which in turn results in a more symmetrical total vertical strain $\left(\epsilon_{y y}^{\text {tot }}\right)$ as shown in Fig. 9(d). Furthermore, as expected, an increase in entry and/or exit strip tension results in a larger total horizontal strain $\left(\epsilon_{x x}^{t o t}\right)$ as shown in Fig. 9(c). The total shear strain fields given in 
Fig. 9(e) show that increasing the tension at the entry results in straightening the material which decreases the bending and shear magnitude in the material. Therefore, all these rich data fields show the expected physical behavior, which is supported by the surface are ratio $\left(A / A_{0}\right)$ given in Fig. 9(f), which remains very close to 1 over all five ROIs. This data can now be used to quantitatively investigate the strip rolling process in more detail than was ever possible before.

\section{Further Improving the Resolution}

From the above analysis, it can be seen that the proposed camera setup is not completely ideal, as the deformation zone is much longer than high and pre-bending already (partially) occurs outside of the FOV. For DIC, sufficient pixels are required in the thickness direction, however, due to the rectangular pixel grid of the camera $(1394 \times 1040)$, this will result in a FOV that does not include the complete length of the deformation zone. Hence, here, an extension of the above proposed t-IDIC methodology is explored in which the full deformation zone is split in multiple side-by-side images captured consecutively with a single camera. Of course, it is possible to correlate these side-by-side image series separately and afterwards combine them into one deformation field, but this will give significant errors at the stitching line(s) because the continuity of the deformation field is not enforced. It is, therefore, preferred to correlate the side-byside images simultaneously to obtain one continuous widefield displacement field with a higher spatial resolution. A significant obstacle is that the rolling velocity is (slightly) different for each image and the pattern can not precisely be traced by just stitching the images.

However, these two obstacles can be avoided in the proposed t-IDIC framework by using a separate velocity corrector for each side-by-side image pair while connecting them to one wide-field deformation field for the different

(a)

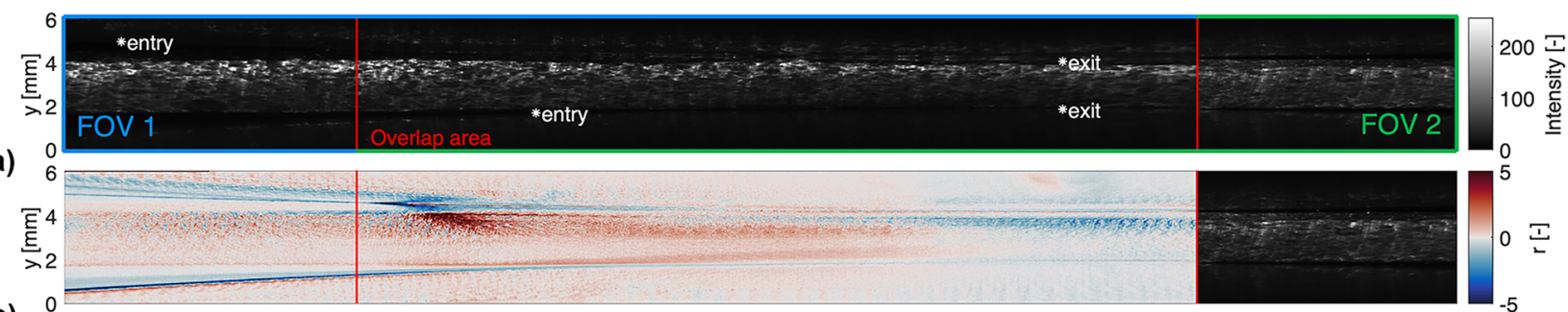

(c)

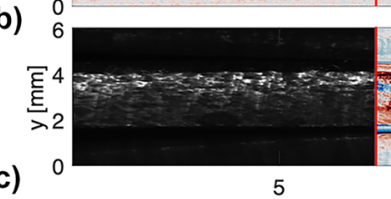

(d)

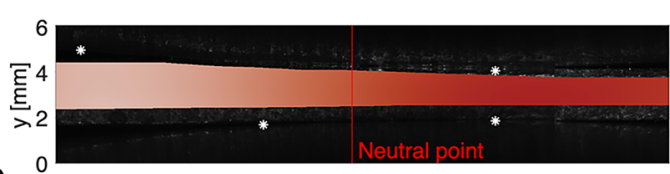

(f)
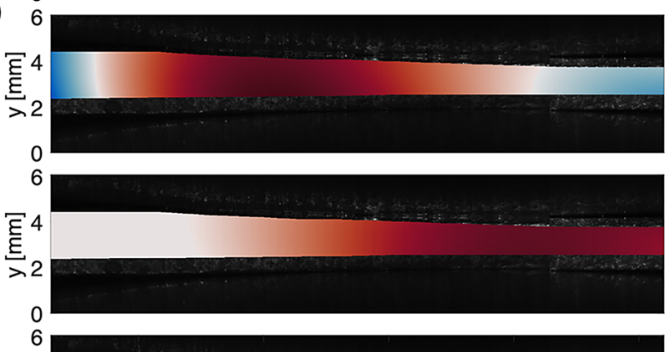

(h)
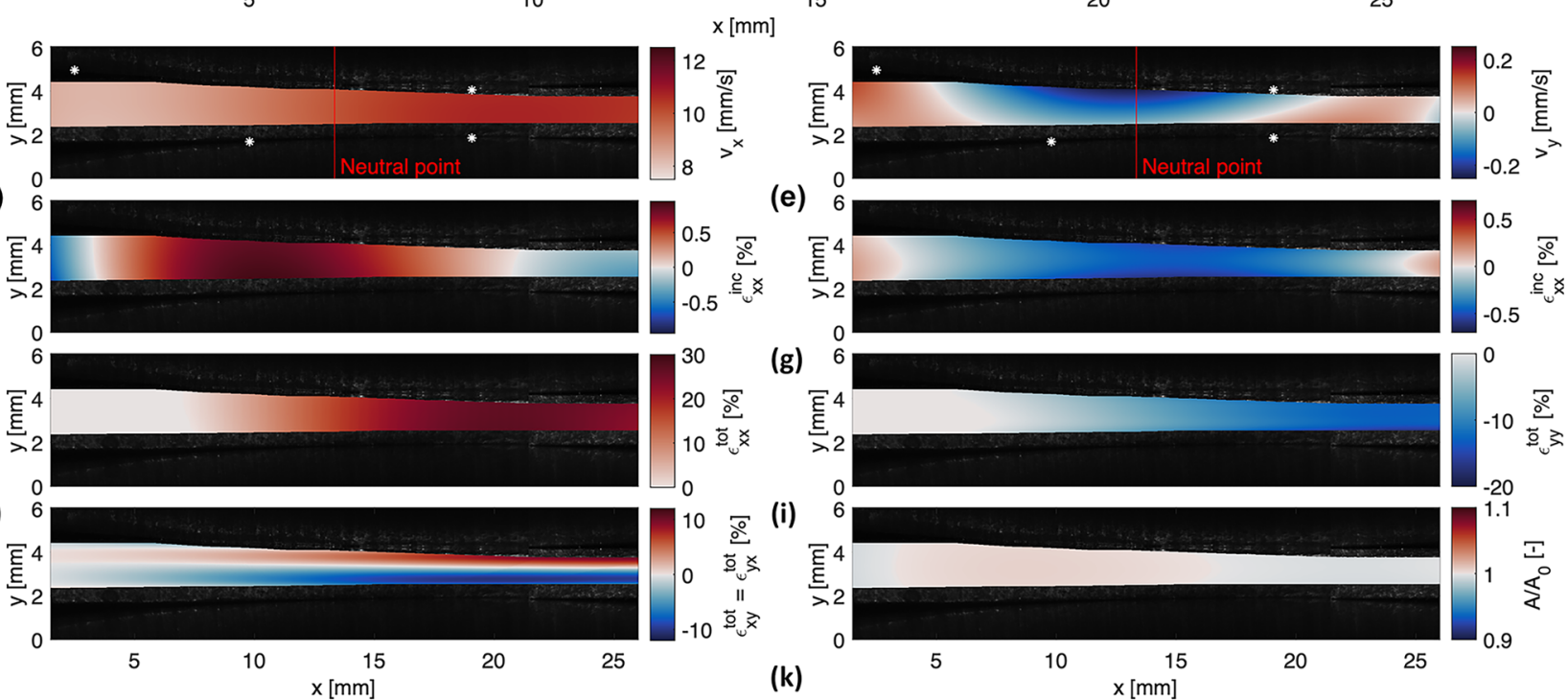

(k)

(e)

(g)

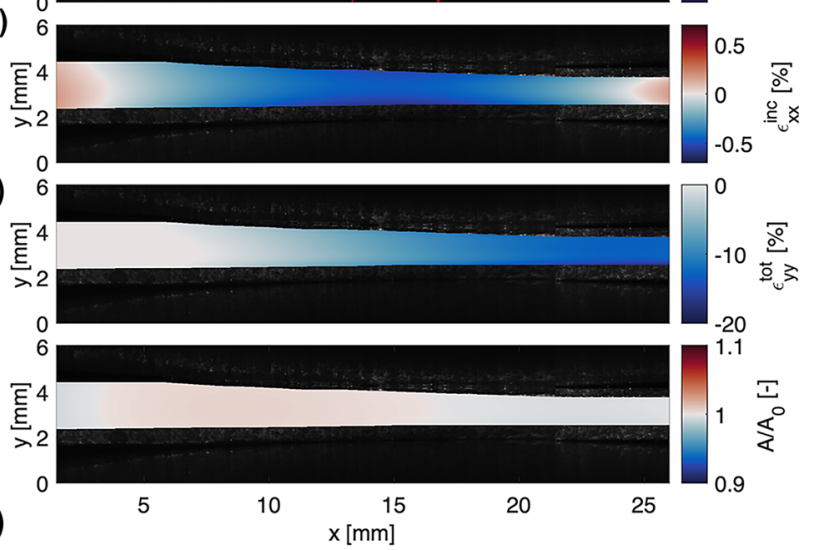

Fig. 10 Improved results for the pilot mill case with (a) the original image reconstructed from two overlapping images with entry and exit points given, (b) the residual field of the front image and (c) the residual field of the back image, (d) the average horizontal velocity $\left(v_{x}\right),(\mathbf{e})$ the average vertical velocity $\left(v_{y}\right),(\mathbf{f})$ the incremental horizontal strain $\left(\epsilon_{x x}^{i n c}\right),(\mathbf{g})$ the incremental vertical strain $\left(\epsilon_{y y}^{i n c}\right),(\mathbf{h})$ the total horizontal strain $\left(\epsilon_{x x}^{\text {tot }}\right)$, (i) the total vertical strain $\left(\epsilon_{y y}^{t o t}\right)$, (j) the total shear strain $\left(\epsilon_{x y}^{\text {tot }}=\epsilon_{y x}^{t o t}\right)$, and $(\mathbf{k})$ the surface area ratio $A / A_{0}$ 
side-by-side image sets. This is studied by capturing the complete ROI using one image series (left ROI) to capture the front and one image series (right ROI) to capture the back of the deformation zone, as shown in Fig. 10(a-c). During the computation of the single deformation field, two residual fields exist, each with their own velocity corrector. If these two sets are correlated by simply adding the extra images, the residual in the overlap region is doubled, giving this area twice the weight, which is unwanted. To solve this issue, in the overlap area, the effect of the residual from the front image is scaled gradually to 0 (i.e. linear in space) while the opposite linear scaling from 0 to 1 is used for the residual of the back image, in the spirit of partition of unity. With this new formulation only the overlap area in the images is needed as input to the extended t-IDIC algorithm to correlate all (e.g. 200) front and back images simultaneously resulting in one wide-field deformation field for the whole process zone. Note that this concept does not need to be limited to two images (i.e. one front and one back image), but can be extended to as many ROIs as deemed necessary, and these ROIs could also be stacked vertically if required.

This novel idea is tested using a work roll force of $750 \mathrm{kN}$ and 10 and $20 \mathrm{kN}$ entry and exit strip tension. In Fig. 10 the results from the correlation are shown. Careful inspection shows that both the average horizontal and vertical velocity field can be slightly better captured than before by taking advantage of the resolution in the strip thickness direction. In comparison to the standard case in Fig. 7, the neutral point stays at virtually the same position, however, since the exit side has been captured more accurately with less acquisition noise present, a more evenly distributed total horizontal and vertical strain fields at the exit are revealed.

\section{Conclusion}

Conventional image correlation algorithms fail at the task of accurate full-field characterization of the velocity and especially the strain fields in the deformation zone during the strip rolling process, due to a combination of harsh environment, non-ideal imaging conditions, high strain rate, poor speckle pattern for image correlation algorithms and unavoidable light reflections. Therefore, in this paper, a novel time-Integrated Digital Image Correlation (t-IDIC) framework has been derived that can handle the abovementioned challenging processing and imaging conditions. The method allows robust and accurate identification of the most important rolling parameters, including, e.g., the neutral point, entry and exit point, and, most importantly, generates high-quality full-field velocity $\left(v_{x}\right.$ and $\left.v_{y}\right)$ and strain $\left(\epsilon_{x x}, \epsilon_{x y}\right.$ and $\left.\epsilon_{y y}\right)$ maps of the deformation zone between the work rolls, as well as important derivatives such as the determinant of the deformation gradient tensor. Therefore, this method enables the validation and falsification of current strip roll models and simulations by analysis of the velocity and strain fields as a function of key input parameters, such as work roll velocity, work roll force and entry and exit strip tension.

The novel t-IDIC algorithm is significantly more accurate and robust than other image correlation algorithms by fully utilizing the knowledge of continuous recurring material motion and by simultaneously correlating multiple (even hundreds) image pairs to obtain one averaged deformation field. Correlation robustness and accuracy is further increased by correcting the deviations in the deformation field between the consecutive images by introducing a unique velocity corrector for each image pair.

The method has been demonstrated on two very different strip rolling cases, i.e. processing of a rubber strip using a small-scale hand-driven mill and a steel strip rolled in a large-scale pilot mill. A validation was done by first determining the best order of the kinematic regularization used to describe the true displacement field, after which the high robustness has been proven by taking out a random image from the image sequence resulting in a negligible error. At last, it was demonstrated that a large number of images is crucial to achieve the very high accuracy in the determination of the displacement fields.

The influence of the work roll force and the strip tension on the obtained full-field data for the pilot mill was then investigated on two proof-of-principle case studies. The velocity fields clearly showed that the rolling process occurs in an asymmetric manner, while the three strain fields provide great quantitative insight into the degree and character of this asymmetry, which was attributed to a non-horizontal feed of the material to the work rolls, because the strip was stored on coils. This was a surprising result, while multiple practical consideration were taken into account, i.e. increased entry and exit strip tension, to realize a horizontal material feed. Additionally, it was found that asymmetric feed can be partially remedied by increasing the entry strip tension and work roll force, resulting in alignment of the strip towards the horizontal axis. Hence, the proposed t-IDIC method was found to be highly sensitive measurement tool for detecting nonsymmetrical deformations. At last, a clear increase in 
distance between the neutral and entry point was found for increasing work roll force.

Finally, as the obtained images in the deformation zone are far from ideal, an improved version of the method is proposed in which the deformation zone is captured using multiple separate (larger magnification) images next to each other instead of one image that covers the complete work roll width, to increase the number of pixels in thickness and width direction. These partial images series are subsequently simultaneously correlated to obtain a continuous deformation field over a larger (high resolution) FOV. A preliminary test revealed that this extension to the t-IDIC would further improve the quality and spatial resolution of the velocity and strain field. In all conclusion, the proposed t-IDIC method enables highly robust and accurate measurement of the full-field behavior of a strip during rolling processes which reveals novel insights in the material behavior and, additionally, the method can easily be adapted and applied to other material flow problems.

\section{Appendix A: One-Step Linearization of the Time-Integrated DIC Framework for Recurring Material Motion}

In this appendix, the minimization of the non-linear brightness conservation equation of the here-proposed time-integrated IDIC framework is derived in a consistent mathematical setting, through a one-step linearization following the work of Neggers et al. [16], in order to arrive at a (modified) GaussNewton iterative optimization scheme that can be easily solved numerically.

The derivation starts from the minimizing (with respect to the DOFs, $\underset{\sim}{\lambda}$ ) of the object function, $\Psi(\underset{\sim}{\underset{\sim}{\lambda}})$, in which the $\mathcal{L}_{2}(\Omega)$-norm of the image residual, $r_{t}(\underline{x})$, is summed over each of the $N_{p x}$ pixels in the region of interest, $\Omega_{s}$, of each of the $t_{d o f}$ images that are simultaneously correlated:

$\left.\underset{\sim}{\lambda_{n} \text { opt }}=\underset{\sim_{n}}{\arg \min }\{\underset{\sim}{\Psi} \underset{\sim}{\lambda})\right\}$

$\Psi(\underset{\sim n}{\lambda})=\sum_{t=1}^{t_{d o f}} \sum_{\Omega_{s}}^{N_{p x}} \frac{1}{2}\left(r_{t}(\underline{x})\right)^{2}$,

where the image residual is the violation of the brightness conservation, which is a function of the position vector $\underline{x}$ :

$r_{t}(\underset{x}{\underline{\lambda}} \underset{\sim}{\lambda})=g_{t-1}(\underline{x})-g_{t}(\underline{x}) \circ \underline{\phi}_{t}(\underset{\sim}{x} \underset{\sim}{\lambda})$.
Here, $\left.\underline{\phi}_{t} \underset{\sim}{\left(\underset{x}{\lambda} \lambda_{n}\right.}\right)$ is the approximate, regularized mapping function of the incremental deformation between consecutive images. As motivated in Sec. 2, the magnitude of the incremental deformation will never be exactly the same for each image pair. Therefore, in the t-IDIC framework, the averaged incremental displacement vector field, $\underline{\delta} u_{a v}(\underline{x})$, which is defined to be equal for all image pairs, is multiplied with a scalar 'velocity corrector', $\alpha_{t}$, that is unique for each image pair, constituting to:

$\underline{\phi}_{t}\left(\underset{x}{x} \underset{\sim_{n}}{\lambda}\right)=\underline{\phi}_{t}\left(\underset{\sim}{x} \underset{\sim_{s}}{\lambda}, \alpha_{t}\right)=\underline{x}+\alpha_{t} \underline{\delta}_{a v}\left(\underset{x}{,} \underset{\sim_{s}}{\lambda}\right)$.

The object function is minimized with respect to all

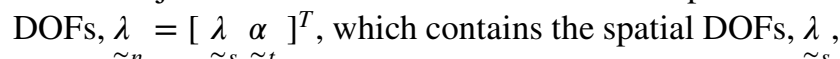
and temporal DOFs, $\alpha_{t}$. The relation between the spatial, temporal, and combined DOFs is defined by, respectively, set $S=\left\{n: 1 \leq n \leq s_{D O F}\right\}$, set $T=\left\{n: s_{D O F}+1 \leq n \leq\right.$ $\left.s_{D O F}+t_{D O F}\right\}$, and set $N=\left\{n: 1 \leq n \leq n_{D O F}\right\}=S \cup T$, with $s_{D O F}, t_{D O F}$ and $n_{D O F}$ being, respectively, the number of spatial, temporal, and combined DOFs. The system of equations is closed by the choice for a spatial regularization of the averages incremental displacement vector field, e.g. the user may choose a polynomial basis:

$\underline{\delta u}_{a v}\left(\underset{x}{\underset{\lambda}{\lambda}} \sim_{n}\right)=\sum_{\chi=1}^{2} \sum_{i=0}^{n} \sum_{j=0}^{m} \lambda_{s, \chi i j} x^{i} y^{j} \underline{e}_{\chi}$.

Minimization of object function, $\underset{\sim}{\Psi(\lambda)} \underset{\sim}{\lambda})$, corresponds to equating its derivative with respect to the DOFs, $\Gamma_{n}(\underset{\sim}{\lambda})$, to zero:

$\forall n \in[N], \Gamma_{n}\left(\underset{\sim n}{\lambda_{\sim}^{o p t}}\right)=\frac{\partial \Psi}{\partial \lambda_{n}}\left(\lambda_{n}^{o p t}\right)=0$.

This non-linear system of equations is linearized and solved using a Gauss-Newton algorithm. During each iteration, $i$, the new DOFs, $\underset{\sim n}{\lambda_{n}^{i+1}}$, is predicted from of the current DOFs, $\underset{\sim}{\lambda_{n}^{i}}$, and the iterative update, $\underset{\sim}{\delta}$, i.e.

$\underset{\sim}{\lambda_{n}^{i+1}}=\underset{\sim_{n}}{\lambda^{i}}+\underset{\sim}{\delta \lambda}$,

resulting in,

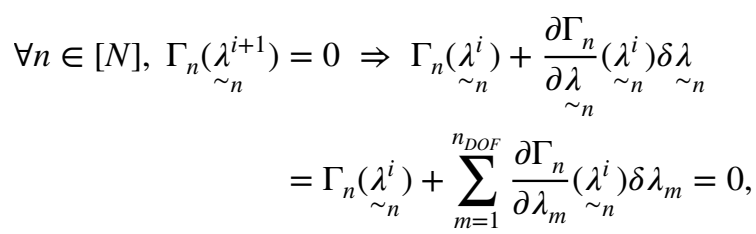

which can be written in matrix form as, 


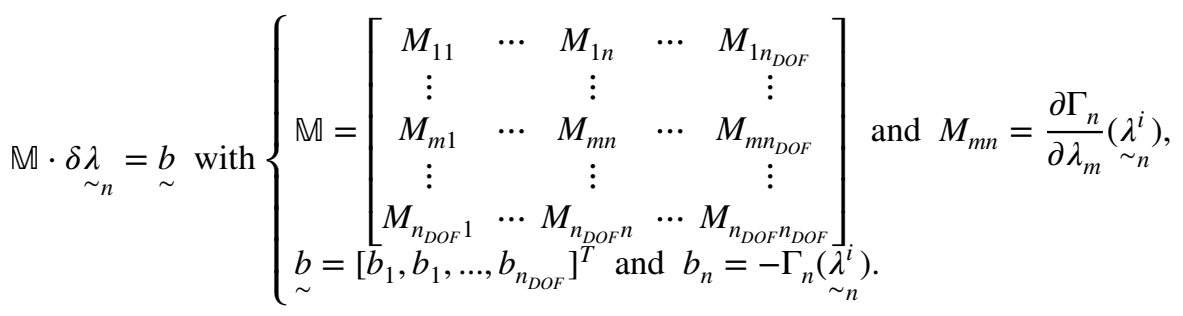

The so-called 'right-hand member', $b$, is given as, $\forall n \in[N]$

$$
\begin{aligned}
& b_{n}=-\Gamma_{n} \underset{\sim n}{\left(\lambda_{n}^{i}\right)}=-\frac{\partial \Psi}{\partial \lambda_{n}}\left(\underset{\sim n}{\lambda_{n}^{i}}\right),
\end{aligned}
$$

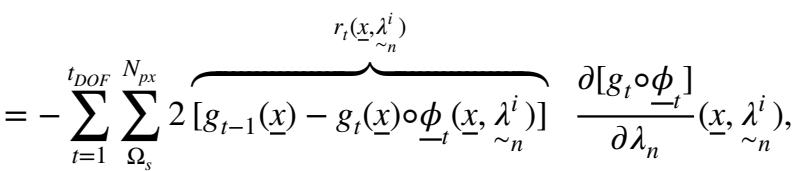

where the chain rule has been applied. The last term can be found by applying the chain rule again:

$\forall n \in[N], \frac{\partial\left[g \circ \underline{\phi}_{t}\right]}{\partial \lambda_{n}}=\underbrace{\operatorname{grad}\left(g_{t}\right) \circ \underline{\phi}_{t}}_{\underline{G}_{t}} \cdot \underbrace{\frac{\partial \underline{\phi}_{t}}{\partial \lambda_{n}}}_{=\underline{\varphi}_{n}^{s t}}$.

Here $\underline{G}_{t}$ is the (true) image gradient of the deformed image, which is often approximated in the literature by the image gradient of the reference image at the expense for correlation robustness, as discussed in detail in Ref. [16], however, here, the (true) image gradient computed at the deformed positions, $\underline{\operatorname{grad}}\left(g_{t}\right) \circ \underline{\phi}_{t}\left(\underset{x}{\underline{x}}{\underset{\sim}{i}}_{n}^{i}\right)$, is used. The second term on the right is the derivative of the mapping function, $\underline{\phi}_{t}$, to the DOFs, which constitutes the set of basis functions, $\underline{\varphi}_{n}$, the form of which depends on whether $\underline{\phi}_{t}$ is derived to $\alpha_{t}$ or $\lambda_{s}$. Therefore, the basis functions are displayed with superscript 'st', i.e. $\underline{\varphi}_{n}^{s t}$, to emphasize the possible spatial or temporal character of the basis functions, i.e.:

$\forall n \in[S], \underline{\varphi}_{n}^{s t}=\frac{\partial \underline{\phi}_{t}}{\partial \lambda_{n}}=\frac{\partial \underline{\phi}_{t}}{\partial \lambda_{s}}=\alpha_{t} \frac{\partial\left(\underline{\delta u}_{a v}\right)}{\partial \lambda_{s}}=\alpha_{t} \underline{\varphi}_{n}^{s}$,

$\forall n \in[T], \underline{\varphi}_{n}^{s t}=\frac{\partial \underline{\phi}_{t}}{\partial \lambda_{n}}=\frac{\partial \underline{\phi}_{t}}{\partial \alpha_{t}}=\left\{\begin{array}{l}\frac{\delta u_{a v} \text { for } n=t,}{0 \text { for } n \neq t,}\end{array}\right.$

where $\underline{\varphi}_{n}^{s}$ are the spatial basis functions (e.g. polynomial functions), resulting in matrix $b$ being written as,

$\forall n \in[N], b_{n}=-2 \sum_{t=1}^{t_{D O F}} \sum_{\Omega_{s}}^{N_{p x}} r_{t}\left(\underset{\sim}{x} \underset{\sim_{n}}{\lambda^{i}}\right) \underline{G}_{t}\left(\underset{\sim}{\left(\lambda_{\sim_{n}}^{i}\right.}\right) \cdot \underline{\varphi}_{n}^{s t}\left(\underset{\sim}{\lambda_{\sim_{n}}}\right)$.
Matrix $\mathbb{M}$ is the derivative of matrix $\underset{\sim}{b}$ to $\underset{\sim}{\lambda}$, i.e. $\mathbb{M}=\frac{\delta b}{\underset{\sim}{\delta \lambda}}$ with $M_{m n}=\frac{\delta b_{n}}{\delta \lambda_{m}}$, this results into the three separate matrices, written as,

$\mathbb{M}=\mathbb{M}^{a}+\mathbb{M}^{b}+\mathbb{M}^{c}$,

which can be rewritten as: $\forall(m, n) \in[N]^{2}$,

$M_{m n}^{a}=-2 \sum_{t=1}^{t_{D O F}} \sum_{\Omega_{s}}^{N_{p x}} \frac{\partial r_{t}}{\partial \lambda_{m}}\left(\underline{x},{\underset{\sim}{\lambda_{n}}}^{i}\right) \underline{G}_{t}\left(\underline{x} \underset{\sim_{n}}{\lambda^{i}}\right) \cdot \frac{\partial \underline{\phi}}{\partial \lambda_{n}}\left(\underline{x} \underset{\sim_{n}}{\lambda^{i}}\right)$,

$M_{m n}^{b}=-2 \sum_{t=1}^{t_{D O F}} \sum_{\Omega_{s}}^{N_{p x}} r_{t}\left(\underset{\sim_{n}}{, \lambda^{i}}\right) \frac{\partial \underline{G}_{t}}{\partial \lambda_{m}}\left(\underset{\sim}{x} \underset{\sim_{n}}{\lambda^{i}}\right) \cdot \frac{\partial \underline{\phi}}{\partial \lambda_{n}}\left(\underset{\sim}{x} \underset{\sim_{n}}{\lambda^{i}}\right)$

$M_{m n}^{c}=-2 \sum_{t=1}^{t_{D O F}} \sum_{\Omega_{s}}^{N_{p x}} r_{t}\left(\underset{\sim}{x} \underset{\sim_{n}}{\lambda^{i}}\right) \underline{G}_{t}\left(\underset{\sim}{x} \underset{\sim_{n}}{\lambda^{i}}\right) \cdot \frac{\partial^{2} \underline{\phi}}{\partial \underline{\lambda}_{m} \partial \lambda_{n}}\left(\underset{\sim}{x} \underset{\sim_{n}}{\lambda^{i}}\right)$

Expanding $\mathbb{M}^{a}$ in the same way as $\underset{\sim}{b}$, yields

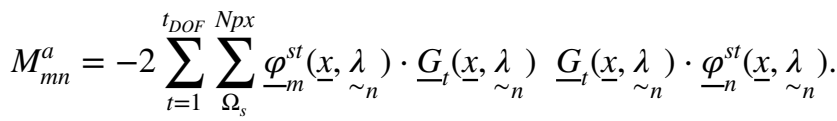

In the second term, $\mathbb{M}^{b}$, the derivative of $\underline{G}_{t}$ with respect to the DOFs changes to, $\forall m \in[N]$,

$\frac{\partial \underline{G}_{t}}{\partial \lambda_{m}}=\underline{\underline{\operatorname{grad}}} \underline{\left.\underline{\operatorname{grad}}\left(g_{t}\right)\right) \circ \underline{\phi}_{t}} \cdot \frac{\partial \underline{\phi}_{t}}{\partial \lambda_{m}}=\underline{\underline{\mathcal{G}}} \cdot \frac{\partial \underline{\phi}_{t}}{\partial \lambda_{m}}$,

resulting in $M_{m n}^{b}$ as,

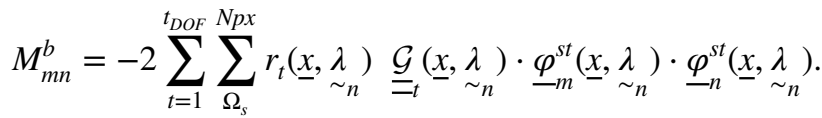

In contrast to a Global Digital Image Correlation framework with a choice for a standard orthogonal kinematic basis, in the t-IDIC framework the shape functions are not linearly independent, therefore, matrix $\mathbb{M}^{c}$ cannot be neglected. This results in the following four cases for the double derivative of the mapping function that is inserted in $\mathbb{M}^{c}$ : 


$$
\begin{aligned}
& \frac{\partial^{2} \underline{\phi}}{\partial \lambda_{n} \partial \lambda_{m}}=0, \\
& \forall m \in[S], \forall n \in[S], \\
& =\left\{\begin{array}{l}
\varphi_{m}^{s} \text { for } n=t, \\
0 \text { for } n \neq t
\end{array}, \quad \forall m \in[S], \forall n \in[T],\right. \\
& =\left\{\begin{array}{l}
\varphi_{n}^{s} \text { for } m=t, \\
0 \text { for } m \neq t
\end{array}, \quad \forall m \in[T], \forall n \in[S],\right. \\
& =0 \text {, } \\
& \forall m \in[T], \forall n \in[T],
\end{aligned}
$$

which concludes the derivation of the iterative GaussNewton optimization scheme for the minimization of the non-linear brightness conservation [16]. Solving Eq. 21 yields the update of $\delta \lambda_{n}$ for each iteration $i$ and, consequently at convergence, the optimal (average) incremental displacement field.

In summary, in the here-proposed t-IDIC framework, both matrices $\mathbb{M}$ and $b$ have been extended to include the added DOFs from the velocity corrector. If, however, only one image pair is correlated, then the t-IDIC framework converges to (the one-step linearized) GDIC framework derived in [16].

\section{Practical considerations:}

First, $\mathbb{M}^{b}$ contains the second gradient of the image, which is highly sensitive to noise, thereby increasing the possibility that the correlation converges at a local minima instead of the global minimum. Therefore, to improve correlation robustness, the choice is made to neglect $\mathbb{M}^{b}$, resulting in a modified Gauss-Newton iterative scheme:

$\mathbb{M}=\mathbb{M}^{a}+\mathbb{M}^{c}$

Second, during strip rolling processes, large deformations are expected, while severe noise is unavoidable due to the processing environment. For similar challenging conditions in terms of convergence, it was demonstrated in [16] that the correlation robustness can be improved by mixing the true image gradient, $\underline{\operatorname{grad}}\left(g_{t}\right) \circ \underline{\phi}_{t}\left(\underline{x}, \underset{\sim_{n}}{\lambda^{i}}\right)$, with the image gradient of the reference image. The rational behind this alteration to the Gauss-Newton framework is that (i) the true image gradient can be written in terms of the back-deformed image, $\tilde{g}_{t}^{i}\left(\underset{\sim}{x} \underset{\sim_{n}}{\lambda^{i}}\right)$, and the incremental deformation gradient tensor, $\underline{\underline{\delta F}}\left(\underline{x}, \underset{\sim_{n}}{\lambda^{i}}\right)$, yielding $\underline{\operatorname{grad}}\left(g_{t}\right) \circ \underline{\phi}_{t}\left(\underline{x}, \underset{\sim_{n}}{\lambda^{i}}\right)=\underline{\operatorname{grad}}\left(\tilde{g}_{t}\right) \cdot \underline{\underline{\delta F^{-T}}}$ and (ii), at each iteration, the back-deformed image converges to the reference image and, therefore, at the converged solution the image gradient of the back-deformed image, $\operatorname{grad}\left(\tilde{g}_{t}^{i}\right)$, should be equal to the image gradient of the reference image, $\operatorname{grad}\left(g_{t-1}\right)$. By combining the true image gradient with the final image gradient at the solution, the truncation error of the linearization of the pattern is reduced, while the noise is further attenuated by the averaging of both image gradients. For this reason, in the here-proposed t-IDIC framework this mixed image gradient is used:
$\underline{G}_{t}\left(\underline{x,}{\underset{\sim}{\lambda_{n}}}^{i}\right) \approx \frac{1}{2}\left(\underline{\operatorname{grad}}\left(g_{t-1}\right)+\underline{\operatorname{grad}}\left(\tilde{g}_{t}^{i}\right)\right) \cdot \underline{\underline{\delta F^{-T}}}\left(\underset{x_{n}}{\lambda_{\sim^{i}}}\right)$.

Acknowledgements The authors acknowledge Daphne van de Giesen and Hans Weel from Tata Steel for preparing and carrying out the pilot mill trials, and Siavash Maraghechi (Eindhoven University of Technology) and Matthijn de Rooij (University of Twente) for proofreading the manuscript.

\section{Declarations}

Conflicts of Interest The authors state that regarding the research and writing of the manuscript, there are no conflicts of interest and that all the authors consent to the content of the manuscript.

Open Access This article is licensed under a Creative Commons Attribution 4.0 International License, which permits use, sharing, adaptation, distribution and reproduction in any medium or format, as long as you give appropriate credit to the original author(s) and the source, provide a link to the Creative Commons licence, and indicate if changes were made. The images or other third party material in this article are included in the article's Creative Commons licence, unless indicated otherwise in a credit line to the material. If material is not included in the article's Creative Commons licence and your intended use is not permitted by statutory regulation or exceeds the permitted use, you will need to obtain permission directly from the copyright holder. To view a copy of this licence, visit http://creativecommons.org/licenses/by/4.0/.

\section{References}

1. Tacke KH, Steffen R (1999) State of steel strip casting. In: Schödter E, Reumer W (eds) Stahl und eisen. Hansebooks, Germany, pp 129-132

2. Ray S (2016) Principles and applications of metal rolling. Cambridge University Press, Cambridge

3. Montmitonnet P, Khalfalla YE, Benyounis KY (2016) Metal Working: Cold Rolling. Reference Module in Materials Science and Materials Engineering, Elsevier

4. Freshwater IJ (1996) Simplified theories of flat rolling I. The calculation of roll pressure, roll force and roll torque. Int J Mech Sci 38(6):633-648

5. Roberts D (1999) Mechanical Principles of Rolling. In: Iron and Steelmaker, USA

6. Hartley P, Sturgess CEN, Liu C, Rowe GW (1989) Experimental and theoretical studies of workpiece deformation, stress, and strain during flat rolling. Int Mater Rev 34(1):19-34

7. Montmitonnet $\mathrm{P}$ (2006) Hot and cold strip rolling processes. Comput Methods Appl Mech Eng 195(48-49):6604-6625

8. Iriarte GE (2011) A new analytical model for cold rolling of sheet and strip. International Journal of Applied Mathematics and Mechanics 7(10):1-50

9. Valjanju DIHPH, Zlitin A (2016) Deformations and velocities during the cold rolling of aluminium alloys. Materiali in tehnologije 50(1):59-67

10. Heydari Vini M, Farhadipour P (2017) Analytical modified model of cold rolling process and investigation of the effect of work roll flattening on the rolling force. Modern Processes in Manufacturing and Production 6(1):5-13

11. Mekicha MA, de Rooij MB, Jacobs L, Matthews DTA, Schipper DJ (2020) Experimental validation of contact models for coldrolling processes. J Mater Process Technol 275 
12. Cowen EA, Monismith SG (1997) A hybrid digital particle tracking velocimetry technique. Exp Fluids 22(3):199-211

13. Keane RD, Adrian R, Zhang Y (1995) Super-resolution particle imaging velocimetry. Meas Sci Technol 6(6):754

14. Zhang P, Peterson SD, Porfiri M (2019) Combined particle image velocimetry/digital image correlation for load estimation. Exp Thermal Fluid Sci 100:207-221

15. Li EB, Tieu AK, Yuen WYD (2003) Application of digital image correlation technique to dynamic measurement of the velocity field in the deformation zone in cold rolling. Opt Lasers Eng 39(4):479-488

16. Neggers J, Blaysat B, Hoefnagels JPM, Geers MGD (2016) On image gradients in digital image correlation. Int $\mathrm{J}$ Numer Meth Eng 105(4):243-260

17. Hild F, Roux S (2012) Comparison of local and global approaches to digital image correlation. Exp Mech 52(9):1503-1519

18. Bergers LIJC, Neggers J, Geers MGD, Hoefnagels JPM (2013) Enhanced global digital image correlation for accurate measurement of microbeam bending. In: Advanced Materials Modelling for Structures. Springer, Berlin, Heidelberg, pp 43-51. ISO 690

19. Cerutti X, Mocellin K, Hassini S, Blaysat B, Duc E (2017) Methodology for aluminium part machining quality improvement considering mechanical properties and process conditions. CIRP J Manuf Sci Technol 18:18-38

20. Rebergue G, Blaysat B, Chanal H, Duc E (2018) Advanced DIC for accurate part deflection measurement in a machining environment. J Manuf Process 33:10-23

21. Van Beeck J, Neggers J, Schreurs PJG, Hoefnagels JPM, Geers MGD (2014) Quantification of three-dimensional surface deformation using global digital image correlation. Exp Mech 54(4):557-570

22. Neggers J, Hoefnagels JPM, Hild F, Roux S, Geers MGD (2014) Direct stress-strain measurements from bulged membranes using topography image correlation. Exp Mech 54(5):717-727

23. Kleinendorst SM, Hoefnagels JPM, Fleerakkers RC, van Maris MPFHL, Cattarinuzzi E, Verhoosel CV, Geers MGD (2016) Adaptive isogeometric digital height correlation: application to stretchable electronics. Strain 52(4):336-354

24. Shafqat S, Van der Sluis O, Geers M, Hoefnagels J (2018) A bulge test based methodology for characterizing ultra-thin buckled membranes. Thin Solid Films 660:88-100

25. Vonk NH, Verschuur NAM, Peerlings RHJ, Geers MGD, Hoefnagels JPM (2020) Robust and precise identification of the hygro-expansion of single fibers: a full-field fiber topography correlation approach. Cellulose 27(12):6777-6792

26. Vanlanduit S, Vanherzeele J, Longo R, Guillaume P (2009) A digital image correlation method for fatigue test experiments. Opt Lasers Eng 47(3-4):371-378

27. Neggers J, Hoefnagels JPM, Geers MGD, Hild F, Roux S (2015) Time resolved integrated digital image correlation. Int J Numer Meth Eng 103(3):157-182

28. Passieux JC, Bouclier R, Périé JN (2018) A space-time PGD-DIC algorithm. Exp Mech 58(7):1195-1206

29. Ruybalid AP, Hoefnagels JPM, van der Sluis O, van Maris MPFHL, Geers MGD (2019) Mixed-mode cohesive zone parameters from integrated digital image correlation on micrographs only. Int J Solids Struct 156:179-193

30. Ruybalid AP, van der Sluis O, Geers MGD, Hoefnagels JPM (2020) Full-field identification of mixed-mode adhesion properties in a flexible, multi-layer microelectronic material system. Eng Fract Mech 226

31. Bigger R, Blaysat B, Boo C, Grewer M, Hu J, Jones A, Klein M, Raghavan K, Reu P, Schmidt T, Siebert T, Simenson M, Turner D, Vieira A, Weikert T (2018) A good practices guide for digital image correlation. International Digital Image Correlation Society, Tech. rep

32. Bornert M, Brémand F, Doumalin P, Dupré JC, Fazzini M, Grédiac M, Hild F, Mistou S, Molimard J, Otreu JJ, Robert L, Surrel Y, Vacher P, Wattrisse B (2009) Assessment of digital image correlation measurement errors: methodology and results. Exp Mech 49(3):353-370

33. Janssen PJM, Hoefnagels JPM, de Keijser TH, Geers MGD (2008) Processing induced size effects in plastic yielding upon miniaturisation. J Mech Phys Solids 56(8):2687-2706

34. Zappa E, Mazzoleni P, Matinmanesh A (2014) Uncertainty assessment of digital image correlation method in dynamic applications. Opt Lasers Eng 56:140-151

35. Jacobs LJM, van Dam KNH, Wentink DJ, de Rooij MB, van der Lugt J, Schipper DJ, Hoefnagels JPM (2021) Effect of Asymmetric Material Entrance in Cold Rolling, Visualized by Integrated Digital Image Correlation (submitted for publication)

Publisher's Note Springer Nature remains neutral with regard to jurisdictional claims in published maps and institutional affiliations. 